\title{
NON-ABELIAN HOPF COHOMOLOGY OF RADFORD PRODUCTS
}

\author{
PHILIPPE NUSS, MARC WAMBST
}

Institut de Recherche Mathématique Avancée, Université de Strasbourg et CNRS, 7, rue René-Descartes, 67084 Strasbourg Cedex, France. e-mail: nuss@math.u-strasbg.fr and wambst@math.u-strasbg.fr

\begin{abstract}
We study the non-abelian Hopf cohomology theory of Radford products with coefficients in a comodule algebra. We show that these sets can be expressed in terms of the non-abelian Hopf cohomology theory of each factor of the Radford product. We write down an exact sequence relating these objects. This allows to compute explicitly the non-abelian Hopf cohomology sets in large classes of examples.
\end{abstract}

MSC 2000 Subject Classification. Primary: 18G50, 16W30 ; Secondary: 14A22, 16S35, 20J06, $55 \mathrm{U} 10$.

Key-words: non-abelian cohomology, Radford products, Hopf comodule algebra, cosimplicial nonabelian groups, Taft algebras.

\section{INTRODUCTION.}

The present article is devoted to the study of the non-abelian Hopf cohomology theory of Radford products. The cohomology theory for Hopf algebras with coefficients in comodule algebras was defined by the authors in [4]. It generalizes the non-abelian cohomology theory for groups ([6], [7]) and is closely related to descent cohomology [3]. Recall that the Radford product of two Hopf algebras (also called in the literature Radford biproducts or bosonization) was introduced in [5] as a generalisation of the semi-direct product of groups (see also Majid's interpretation in terms of braided categories [2]).

The Radford product $H \star E$, which is a Hopf algebra, is defined for a Hopf algebra $H$ and any algebra $E$ in the braided Yetter-Drinfeld category over $H$ [11]. Our main result allows to describe the cohomology sets of $H \star E$ in terms of those of $H$ and of a braided version of the cohomology sets of $E$. This decomposition has a counterpart in the non-abelian cohomology theory of a semi-direct product of groups. We now detail the organisation of the article.

In Section 0, we write down our conventions, for instance the diagrammatic language of YetterDrinfeld categories that we abundantly make use of. Section 1 is devoted to the Radford products. We briefly recall their definition and point out some properties of their comodule algebras. In particular we show that a comodule algebra structure over a Radford product $H \star E$ is equivalent to the datum of two compatible $H$ - and $E$-comodule algebra structures (Proposition 1.1).

Reminders on the non-abelian Hopf cohomology theory $\mathcal{H}^{*}(H, F)$ of a Hopf algebra $H$ with coefficients in a comodule algebra $F$ are given in Section 2. There we extend essentially in two ways the definition introduced in [4] in terms of pre-cosimplicial algebras. Firstly we show that any functor $\mathbf{G}$ defined on a suitable subcategory of the category of algebras with values in the latter gives rise to a new non-abelian Hopf cohomology theory $\mathcal{H}^{*} \mathbf{G}(H, F)$. Secondly we construct a theory $\mathcal{H}_{\star}^{*}(E, F)$ by adapting the definitions when the Hopf algebra $E$ belongs to the braided Yetter-Drinfeld category of $H$. The main result (Theorem 2.2) expresses $\mathcal{H}^{*}(H \star E, F)$ in terms of $\mathcal{H}^{*}(H, F)$ and $\mathcal{H}_{\star}^{*}(E, F)$. We moreover show that the 1-cohomology set $\mathcal{H}^{1}(H \star E, F)$ fits into an exact sequence of pointed sets

$$
\mathcal{H}^{1} \mathbf{C}_{E}(H, F) \longrightarrow \mathcal{H}^{1}(H \star E, F) \longrightarrow \mathcal{H}_{\star}^{1}(E, F)^{H}
$$


where the first map is injective (Theorem 2.4). The left-hand side term is the 1-cohomology set associated with the "E-coinvariant" functor $\mathbf{C}_{E}=(-)^{E}$, and the right-hand side term is the $H$-coinvariant subset of $\mathcal{H}_{\star}^{1}(E, F)$ in a sense we define in the core of the text.

Section 3 is devoted to the computation of some examples. We begin with the case where the coefficient comodule algebra $F$ is equal to $E$. In Proposition 3.1 we show that $\mathcal{H}^{0}(H \star E, E)$ is equal to the group of units of $k$, and that $\mathcal{H}^{1}(H \star E, E)$ is in bijection with the set of groupoidal elements in $H$. We specify this example when $H \star E$ is a Taft algebra (Corollary 3.2). We also study the example when $H$ and $E$ are Hopf algebras of functions over groups (Proposition 3.3). Finally, Section 4 is a foray into the world of groups. We describe the non-abelian cohomology sets of a semi-direct product of two groups (Proposition 4.1) and obtain a similar decomposition as that stated in Theorem 2.2 .

\section{Conventions and prerequisites.}

Let $k$ be a fixed commutative and unital ring. By a $k$-module we understand a symmetric $k$-bimodule. The unadorned symbol $\otimes$ between two $k$-modules stands for $\otimes_{k}$. By (co-)algebra we mean a (co-)unital (co-)associative $k$-(co-)algebra. By (co-)module over a (co-)algebra $E$, we always understand a right $E$-(co-)module unless otherwise stated. Let $M$ be a $k$-module. We identify in a systematic way $M \otimes k$ with $M$. For any algebra $F$, we denote by $F^{\times}$the group of invertible elements in $F$.

Let $H$ be a Hopf algebra with multiplication $\mu_{H}$, unit map $\eta_{H}$, comultiplication $\Delta_{H}$, counit map $\varepsilon_{H}$, and antipode $\sigma_{H}$. To denote the coactions on elements, we use the Sweedler-Heyneman convention, that is for any Hopf algebra $H$, the coproduct $\Delta_{H}(h)$ of an element $h \in H$ is denoted by $h_{1} \otimes h_{2}$ (notice the omission of the summation sign). If $M$ is an $H$-comodule with coaction $\varrho_{M, H}$, for any $m \in M$ we set $\varrho_{M, H}(m)=m_{0} \otimes m_{1}$. From now on, we implicitely mean that $H$ is an $H$-module by $\mu_{H}$ and an $H$-comodule by $\Delta_{H}$.

In the sequel we shall use the diagrammatic language for morphisms (see for example [11]). The pictorial conventions are given in Figure 0.1.

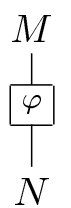

A morphim of modules $\varphi: M \rightarrow N$

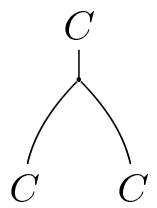

The comultiplication of a coalgebra $C$

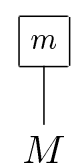

The element $m$ in a module $M$

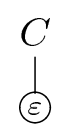

The counit of a coalgebra $C$

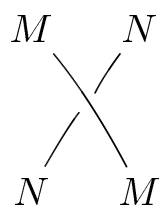

The flip between the modules $M$ and $N$

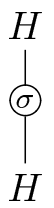

The antipode of a Hopf algebra $H$

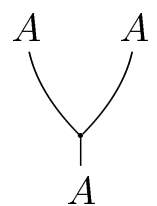

The multiplication of an algebra $A$

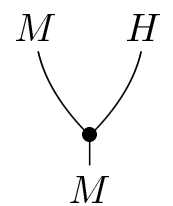

The action of a Hopf algebra $H$ on a module $M$
(7)

A

The unit of an algebra $A$

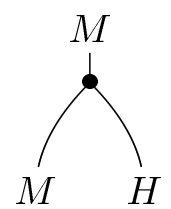

The coaction of a Hopf algebra $H$ on a comodule $M$ 
Notice that an element $m$ of a module $M$ is identified with the $k$-linear map from $k$ to $M$ sending 1 to $m$. Hence that element is pictorially represented by a box without input and with $M$ as output. Recall that, given two $k$-modules $M$ and $N$, the flip is the map from $M \otimes N$ to $N \otimes M$ sending $m \otimes n$ to $n \otimes m$, for any $m \in M$ and $n \in N$.

The tensor product $M \otimes M^{\prime}$ of two $H$-modules $M$ and $M^{\prime}$ becomes an $H$-module via the action given by $\left(m \otimes m^{\prime}\right) \cdot h=m \cdot h_{1} \otimes m^{\prime} \cdot h_{2}$. Here $m$ belongs to $M, m^{\prime}$ to $M^{\prime}$, and $h$ to $H$. Dually, the tensor product $N \otimes N^{\prime}$ of two $H$-comodules $N$ and $N^{\prime}$ is an $H$-comodule through the coaction $\varrho_{N \otimes N^{\prime}, H}$ defined on $n \otimes n^{\prime} \in N \otimes N^{\prime}$ by $\varrho_{N \otimes N^{\prime}, H}\left(n \otimes n^{\prime}\right)=n_{0} \otimes n_{0}^{\prime} \otimes n_{1} n_{1}^{\prime}$.

Let $M$ still be an $H$-module and $N$ be an $H$-comodule. In [11], Yetter defines a map $\tau_{M, N}: M \otimes N \longrightarrow N \otimes M$ given, on any indecomposable tensor $m \otimes n$ in $M \otimes N$, by $\tau_{M, N}(m \otimes n)=$ $n_{0} \otimes m \cdot n_{1}$. We shall use the following diagrammatic notation for $\tau_{M, N}$ :

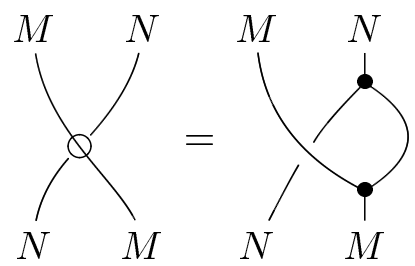

The definition of the map $\tau_{M, N}$

- Figure $0.2-$

It is known that the map $\tau_{M, N}$ is a pre-braiding [11]. This means that the four equalities drawn down in Figure 0.3 hold, when $M$ and $M^{\prime}$ are $H$-modules, $N$ and $N^{\prime}$ are $H$-comodules, $\varphi: M \longrightarrow M^{\prime}$ is a morphism of $H$-modules, and $\psi: N \longrightarrow N^{\prime}$ is a morphism of $H$-comodules.

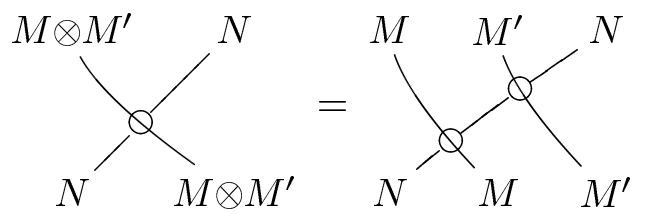

Relation 0.3.a

Compatibility of $\tau_{-, N}$ with $\otimes$

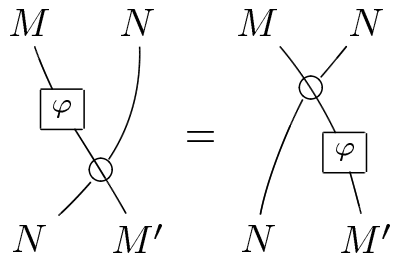

Relation 0.3.c

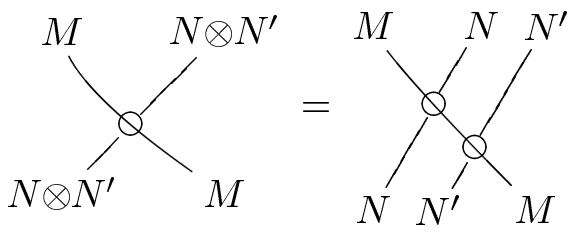

Relation 0.3.b

Compatibility of $\tau_{M,-}$ with $\otimes$

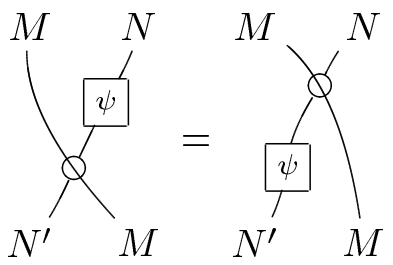

Relation 0.3.d

$\tau_{M, N^{\prime}} \circ\left(\operatorname{id}_{M} \otimes \psi\right)=\left(\psi \otimes \operatorname{id}_{M}\right) \circ \tau_{M, N}$

- Figure 0.3 - 
Moreover, for any $H$-module $M$ and any $H$-comodule $N$, one easily shows the relations drawn in Figure 0.4. We shall need them frequently in the sequel.

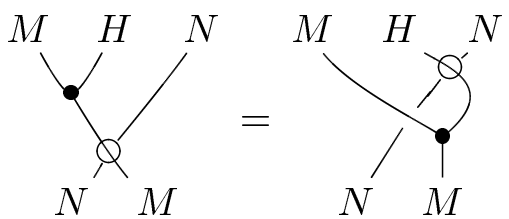

Relation 0.4.a

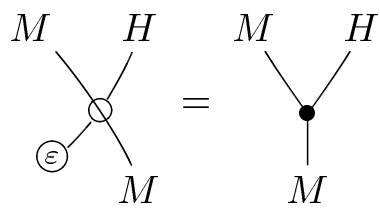

Relation 0.4.c

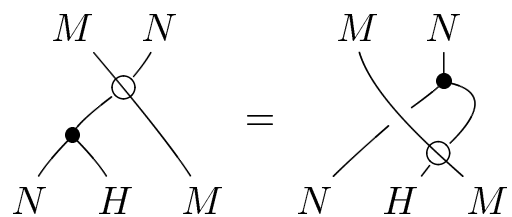

Relation 0.4.b

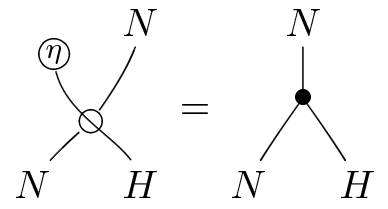

Relation 0.4.d

- Figure $0.4-$

The Yetter-Drinfeld category $\mathscr{Y} \mathscr{D}_{H}^{H}$ of a Hopf algebra $H$ is defined in the following way ([11]). Its objects are the $k$-modules $M$ which are both $H$-modules and $H$-comodules subject to the compatibility law $m_{0} \cdot h_{1} \otimes m_{1} h_{2}=\left(m \cdot h_{2}\right)_{0} \otimes h_{1}\left(m \cdot h_{2}\right)_{1}$, where $m \in M$ and $h \in H$. The latter relation is drawn in Figure 0.5.

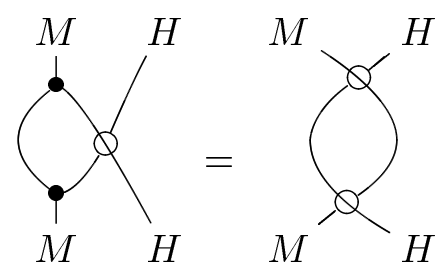

- Figure 0.5 -

Assumption : From now on and to the end of the article, we suppose that $H$ is a Hopf algebra such that the co-opposite algebra of $H$ is a Hopf algebra.

Under this assumption, the pre-braiding $\tau_{-,-}$is a braiding and $\left(\mathscr{Y}_{H}^{H}, \otimes\right)$ is a braided strict monoidal category [11]. Remark that the comodule structure of the tensor product $M \otimes N$ of two $H$-comodules $M$ and $N$ is given by the formula $\varrho_{M \otimes N, H}=\left(\operatorname{id}_{M} \otimes \tau_{H, N}\right) \circ\left(\varrho_{M, H} \otimes \operatorname{id}_{N}\right)$, summarized in Figure 0.6.

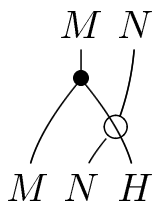

The H-comodule structure on $M \otimes N$

- Figure 0.6 - 
Lemma 0.1. Let $H$ be a Hopf algebra. Let $E$ be a bialgebra in the category $\mathscr{Y} \mathscr{D}_{H}^{H}$ and let $N$ be an $H$-comodule. The map $\tau_{E, N}: E \otimes N \longrightarrow N \otimes E$ defined by Figure 0.2 is a morphism of $H$-comodules.

Proof: The proof is entirely contained in the pictorial computation of Figure 0.7.
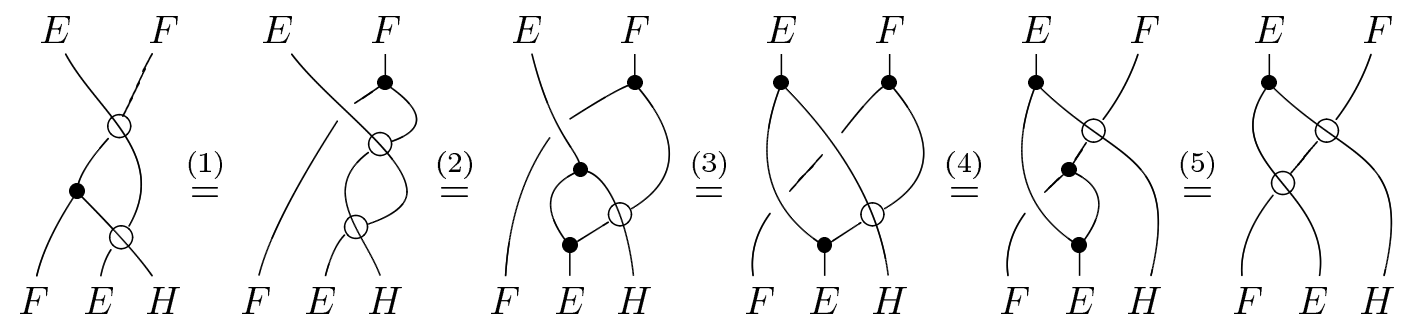

- Figure 0.7 -

The first and the fourth equalities are consequences of Relation 0.4.b. The second one follows from the Yetter-Drinfeld compatibility (Figure 0.5). The third one is an obvious property of the flip, whereas the fifth is the convention adopted in Figure 0.2 .

For any bialgebra $E$ in the category $\mathscr{Y} \mathscr{D}_{H}^{H}$, we define an algebra $F$ to be a Radford $E$-comodule algebra if $F$ is both an $H$-comodule (pictorially $\boldsymbol{R}$ ) and an $E$-comodule (pictorially $\boldsymbol{R}$ ), such that the three conditions drawn in Figure 0.8 are satisfied.

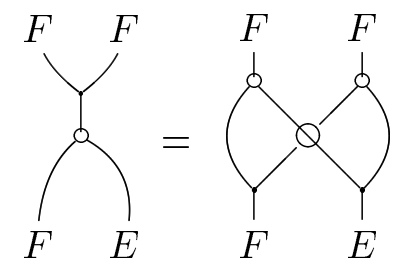

Relation 0.8.a

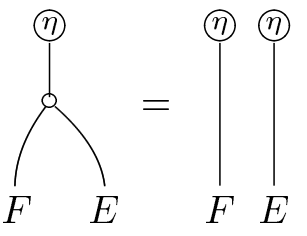

Relation 0.8.b

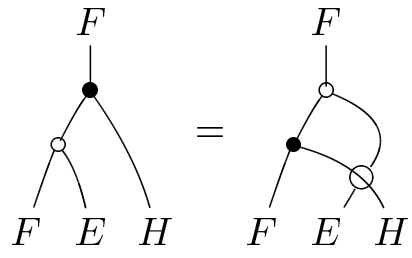

Relation 0.8.c

- Figure $0.8-$

In particular, any $E$-comodule algebra $F$ in $\mathscr{Y} \mathscr{D}_{H}^{H}$ - for example $E$ itself - is a Radford $E$-comodule algebra. Indeed, the two first relations are consequences of the $E$-comodule structure of $F$. The third one is the transcription of the fact that $\varrho_{F, E}$ is a morphism of $H$-comodules. We choose the name Radford comodule algebra in view of the results obtained in Proposition 1.1 of the next section.

\section{Radford products}

1.1. Some definitions. Recall that, for any bialgebra $E$ in $\mathscr{Y} \mathscr{D}_{H}^{H}$, one may construct the Radford product $H \star E$ ([5], [1]). It is a bialgebra and contains $H$ as a sub-bialgebra and $E$ as a subalgebra. As a module, $H \star E$ is equal to $H \otimes E$. The product, coproduct, unit, and counit are respectively given on $h \star x$ and $h^{\prime} \star x^{\prime}$ in $H \star E$ by

$$
\begin{array}{cl}
(h \star x)\left(h^{\prime} \star x^{\prime}\right)=h h_{1}^{\prime} \star\left(x \cdot h_{2}^{\prime}\right) x^{\prime} & \Delta_{H \star E}(h \star x)=\left(h_{1} \star\left(x_{1}\right)_{0}\right) \otimes\left(h_{2}\left(x_{1}\right)_{1} \star x_{2}\right) \\
1_{H \star E}=1_{H} \star 1_{E} & \varepsilon_{H \star E}(h \star x)=\varepsilon_{H}(h) \varepsilon_{E}(x) .
\end{array}
$$


The diagrammatic transcription of the two first formulas is drawn in Figure 1.1.

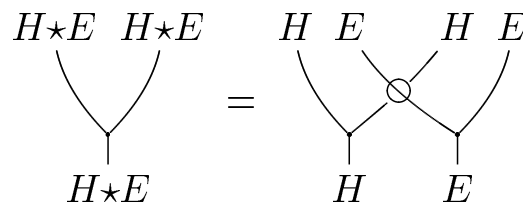

The multiplication of $H \star E$

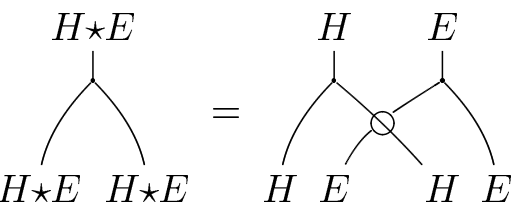

The comultiplication of $H \star E$

- Figure 1.1 -

The canonical projections and inclusions are summarised in the following diagram:

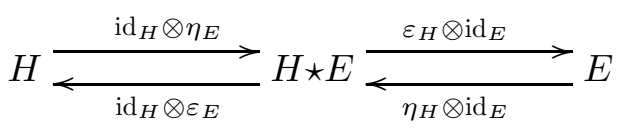

The two left maps are morphisms of bialgebras, whereas in general $\eta_{H} \otimes \mathrm{id}_{E}$ is a only a map of algebras and $\varepsilon_{H} \otimes \mathrm{id}_{E}$ is only a map of coalgebras.

We also recall that, if $E$ is a Hopf algebra in $\mathscr{Y} \mathscr{D}_{H}^{H}$, one may turn $H \star E$ into a Hopf algebra by using the map given by Figure 1.2 as an antipode [5].

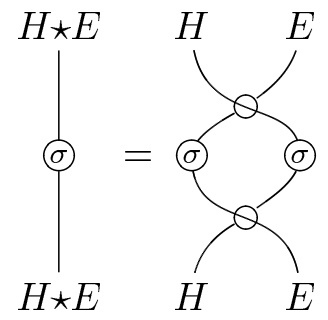

The antipode of $H \star E$

- Figure 1.2 -

When $E$ is trivial both as an $H$-module and as an $H$-comodule, then $H \star E$ is simply the tensor product $H \otimes E$ of the two Hopf algebras $H$ and $E$.

\subsection{Comodule algebras over Radford products.}

Proposition 1.1. Let $H$ be a Hopf algebra. Let $E$ be a bialgebra in $\mathscr{Y} \mathscr{D}_{H}^{H}$ and $F$ be an algebra. The two following statements are equivalent:

1) $F$ is an $H \star E$-comodule algebra ;

2) $F$ is an $H$-comodule algebra and $F$ is a Radford $E$-comodule algebra.

Proof: First suppose that $F$ is an $H \star E$-comodule algebra. Let $\varrho_{F, H \star E}: F \longrightarrow F \otimes(H \star E)$ be the coaction of $H \star E$ on $F$ (pictorially $\$ ). Define $\varrho_{F, H}: F \longrightarrow F \otimes H$ and $\varrho_{F, E}: F \longrightarrow F \otimes E$ by

$$
\varrho_{F, H}=\left(\operatorname{id}_{F} \otimes \operatorname{id}_{H} \otimes \varepsilon_{E}\right) \circ \varrho_{F, H \star E} \quad \text { and } \quad \varrho_{F, E}=\left(\operatorname{id}_{F} \otimes \varepsilon_{H} \otimes \operatorname{id}_{E}\right) \circ \varrho_{F, H \star E}
$$

(pictorially (e) and ).

The fact that $F$ is an $H \star E$-comodule is represented in Figure 1.3: 


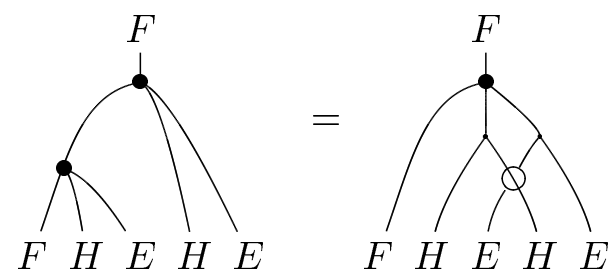

- Figure 1.3 -

We apply the counit maps $\varepsilon_{H}$, respectively $\varepsilon_{E}$, at the two corresponding outputs of the equality in Figure 1.3. Using the defining axiom of the counit map and the relations 0.3.c and 0.3.d of Figure 0.3, one gets the four relations of Figure 1.4.

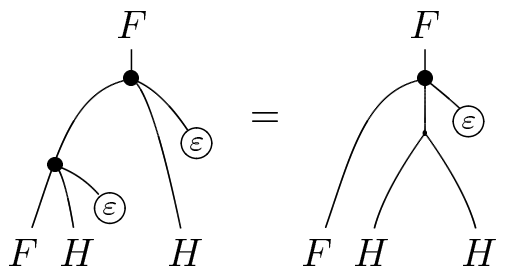

Relation 1.4.a

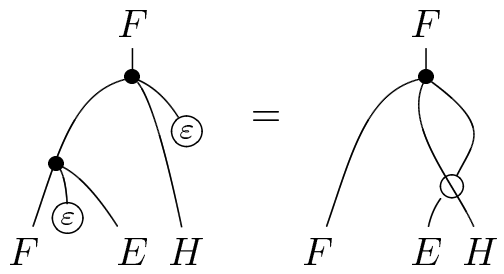

Relation 1.4.c

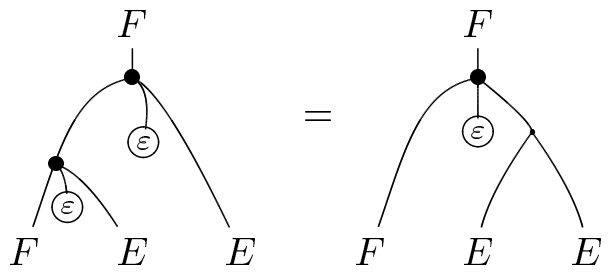

Relation 1.4.b

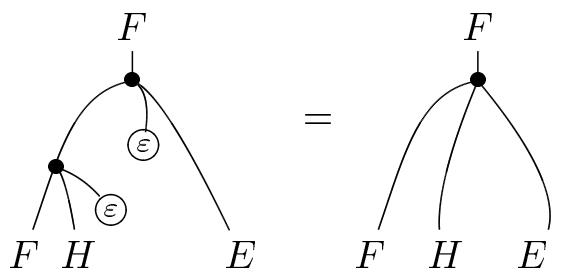

Relation 1.4.d

- Figure $1.4-$

The two upper relations show that $\varrho_{F, H}$, respectively $\varrho_{F, E}$, endow $F$ with an $H$-, respectively $E$-, comodule structure (in both cases, the axiom $\left(\mathrm{id}_{F} \otimes \varepsilon_{-}\right) \circ \varrho_{F,-}=\mathrm{id}_{F}$ is obviously satisfied). The two lower equalities imply the compatibility relation 0.8.c of Figure 0.8. Moreover, Relation 0.8.b is implied by $\varrho_{F,-} \circ \eta_{F}=\eta_{F} \otimes \eta_{-}$, which is obvious.

Consider now Figure 1.5, which depicts that $\varrho_{F, H \star E}$ is a morphism of algebras.

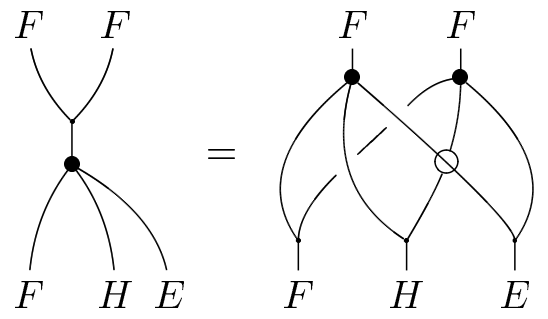

- Figure $1.5-$ 
Apply the counit map $\varepsilon_{E}$ to Figure 1.5. Using the fact that $\varepsilon_{E}$ is a morphism of algebras and verifies Relation 0.3.c, one gets the following equality, which proves that $\varrho_{F, H}$ is a morphism of algebras:

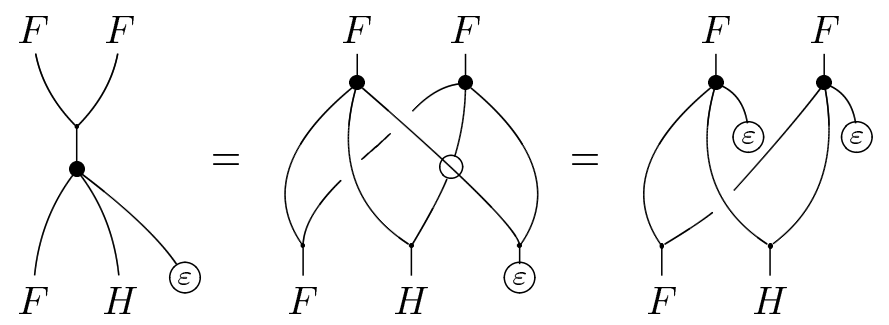

- Figure $1.6-$

Similarly, apply the counit map $\varepsilon_{H}$ to Figure 1.5. Using the fact that $\varepsilon_{H}$ is a morphism of algebras and verifies Relations 0.4.c and 1.4.c, one obtains Relation 0.8.a for $\varrho_{F, E}$.
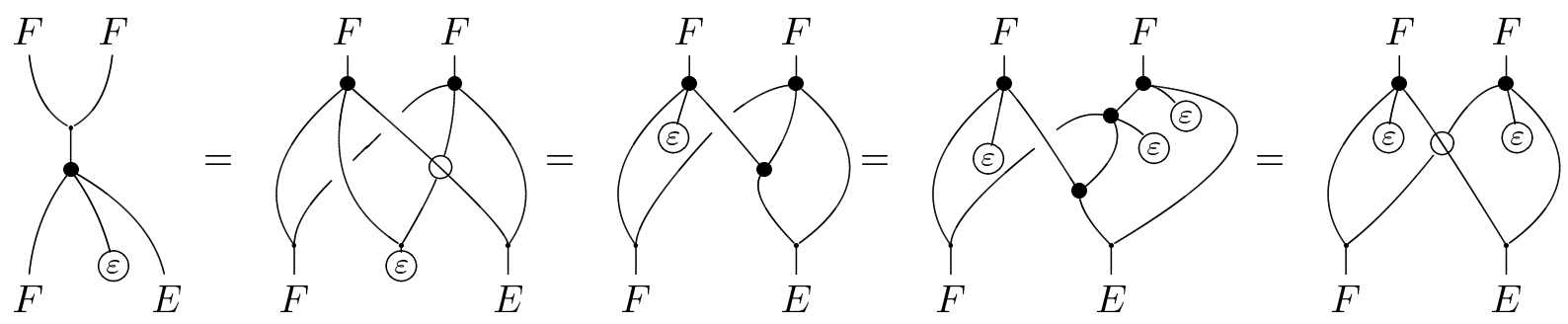

- Figure 1.7 -

This achieves the proof of $1 \Longrightarrow 2$.

Conversely, take an $H$-comodule algebra $F$ (with coaction $\varrho_{F, H}$ ) which is also a Radford $E$-comodule algebra (via the coaction $\left.\varrho_{F, E}\right)$. We show that the map $\varrho_{F, H \star E}: F \longrightarrow F \otimes(H \star E)$ defined by

$$
\varrho_{F, H \star E}=\left(\varrho_{F, H} \otimes \operatorname{id}_{E}\right) \circ \varrho_{F, E}
$$

endows $F$ with a structure of $H \star E$-comodule algebra.

We obviously have the identity $\left(\mathrm{id}_{F} \otimes \varepsilon_{H \star E}\right) \circ \varrho_{F, H \star E}=\mathrm{id}_{F}$. The coassociativity of $\varrho_{F, H \star E}$ results from the equalities 1.8.a of Figure 1.8 below. It is an immediate consequence of the compatibility relation 0.8.c of Figure 0.8 and of the definition of the comultiplication of $H \star E$. The fact that $F$ is an $H \star E$-comodule algebra follows from the obvious relation $\varrho_{F, H \star E} \circ \eta_{F}=\eta_{F} \otimes \eta_{H \star E}$ and from the equalities 1.8.b. There one uses the axiom 0.8.a of Figure 0.8, the $H$-comodule algebra structure of $F$, and Relation 0.4 .b of Figure 0.4 . 


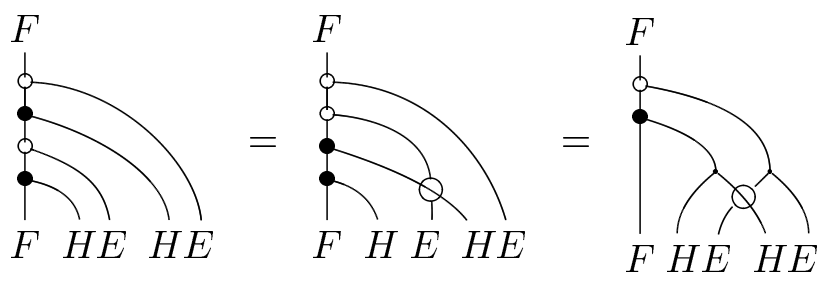

1.8.a

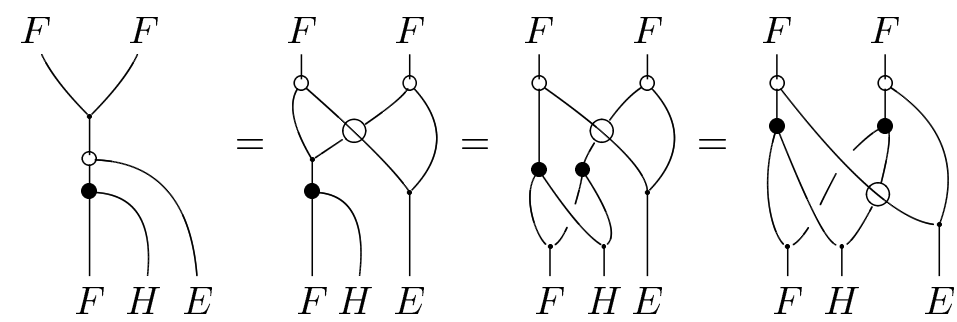

1.8.b

- Figure $1.8-$

\subsection{Radford extensions of scalars.}

Proposition 1.2. Let $H$ be a Hopf algebra. Let $E$ be a bialgebra in $\mathscr{Y} \mathscr{D}_{H}^{H}$ and $F$ be a Radford $E$-comodule algebra. The module $F \otimes E$ is a Radford E-comodule algebra for the multiplication given by Relation 1.9.a, the unit $\eta_{F} \otimes \eta_{E}$, the $H$-coaction map depicted in Relation 1.9.b, and the E-comodule map $\operatorname{id}_{F} \otimes \Delta_{E}$.

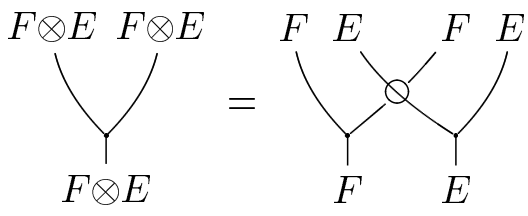

Relation 1.9.a

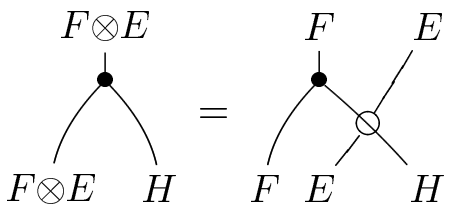

Relation 1.9.b

- Figure 1.9 -

Proof: Using the diagrammatic language, the Reader may check all the axioms.

The Radford $E$-comodule algebra of Proposition 1.2 will be denoted by $F \star E$. One iterates the construction in order to build $(F \star E) \star E$. The latter Radford $E$-comodule algebra will be denoted for simplicity by $F \star E \star E$.

Remark 1.3. : The coaction map $\varrho_{F, E}: F \longrightarrow F \star E$ is a morphism of $H$-comodules by Relation 0.8.c in Figure 0.8. 


\section{Radford products and non-abelian Hopf cohomology theory.}

In this section, we recall and generalize the construction of the non-abelian Hopf cohomology theory developped in [4]. We study the case when the Hopf algebra is a Radford product.

2.1. Remainders on non-abelian Hopf cohomology theory. By a pre-cosimplicial object in a category $\mathscr{C}$, we understand a diagram $\mathcal{A}$ of the type

$$
\mathcal{A}=A^{0} \stackrel{d^{0}}{\stackrel{d^{1}}{\longrightarrow}} A^{1} \stackrel{\frac{d^{0}}{\stackrel{d^{1}}{d^{2}}}}{\longrightarrow} A^{2},
$$

where $A^{i}$ is an object in $\mathscr{C}$ and $d^{j}$ are morphisms in $\mathscr{C}$ such that the pre-cosimplicial relations $d^{i} d^{j}=d^{j} d^{i-1}$ hold for $i>j$.

Suppose now that $\mathscr{C}$ is the category of groups. The non-abelian 0-cohomology group $\mathbb{H}^{0}(\mathcal{A})$ of a pre-cosimplicial group $\mathcal{A}$ is the equalizer of the pair $\left(d^{0}, d^{1}\right)$ :

$$
\mathbb{H}^{0}(\mathcal{A})=\left\{x \in A^{0} \mid d^{1}(x)=d^{0}(x)\right\} .
$$

The non-abelian 1-cohomology pointed set $\mathbb{H}^{1}(\mathcal{A})$ of $\mathcal{A}$ is the right quotient

$$
\mathbb{H}^{1}(\mathcal{A})=A^{0} \backslash \mathbb{Z}^{1}(\mathcal{A})
$$

where the set $\mathbb{Z}^{1}(\mathcal{A})$ of 1 -cocycles is the subset of $A^{1}$ defined by

$$
\mathbb{Z}^{1}(\mathcal{A})=\left\{X \in A^{1} \mid d^{2}(X) d^{0}(X)=d^{1}(X)\right\}
$$

The group $A^{0}$ acts on the right on $A^{1}$ by

$$
X<x=\left(d^{1} x^{-1}\right) X\left(d^{0} x\right),
$$

where $X \in A^{1}$ and $x \in A^{0}$. This action restricts to $\mathbb{Z}^{1}(\mathcal{A})$. Two 1-cocycles $X$ and $X^{\prime}$ are cohomologous if they belong to the same orbit under this action. The quotient set $\mathbb{H}^{1}(\mathcal{A})=$ $A^{0} \backslash \mathbb{Z}^{1}(\mathcal{A})$ is pointed with distinguished point the class of the neutral element of $A^{1}$.

Let $H$ be a Hopf algebra and $F$ be an $H$-comodule algebra with multiplication $\mu_{F}$ and coaction $\varrho_{F, H}$. In the spirit of [9], we defined in [4] two maps $d^{i}: F \longrightarrow F \otimes H(i=0,1)$ and three maps $d^{i}: F \otimes H \longrightarrow F \otimes H \otimes H(i=0,1,2)$ by the formulae

$$
\begin{aligned}
& d^{0}(x)=\varrho_{F, H}(x), \quad \quad d^{1}(x)=x \otimes 1, \\
& d^{0}(X)=\left(\varrho_{F, H} \otimes \operatorname{id}_{H}\right)(X), \quad d^{1}(X)=\left(\operatorname{id}_{F} \otimes \Delta_{H}\right)(X), \quad d^{2}(X)=X \otimes 1,
\end{aligned}
$$

where $x \in F$ and $X \in F \otimes H$. The maps $d^{i}$ are easily seen to be morphisms of algebras. The diagram $\mathcal{C}(H, F)$ given by

$$
F \stackrel{d^{0}}{\stackrel{d^{1}}{\longrightarrow}} F \otimes H \underset{\stackrel{d^{2} \longrightarrow}{\longrightarrow}}{\stackrel{\frac{d^{0}}{\longrightarrow}}{\longrightarrow}} F \otimes H \otimes H
$$

is a pre-cosimplicial object in the category of algebras. In the sequel the diagram $\mathcal{C}(H, F)$ lives in fact in a certain subcategory $\mathcal{B}$ of the category of algebras, for instance a category of comodule algebras. 
Let $\mathbf{G}$ be a functor from the category $\mathcal{B}$ to the category of algebras. The diagram $\mathcal{C}(H, F)$ gives rise to a pre-cosimplicial diagram $\mathcal{C}^{\times} \mathbf{G}(H, F)$ in the category of groups by setting:

$$
\mathcal{C}^{\times} \mathbf{G}(H, F)=\mathbf{G}(F) \times \underset{d^{1}}{\stackrel{d^{0}}{\longrightarrow}} \mathbf{G}(F \otimes H)^{\times} \underset{\stackrel{d^{2}}{\stackrel{d^{1}}{\longrightarrow}}}{\longrightarrow} \mathbf{G}(F \otimes H \otimes H)^{\times}
$$

(we still denote by $d^{i}$ the restrictions of the maps $\mathbf{G}\left(d^{i}\right)$ to invertible elements). The non-abelian Hopf cohomology theory $\mathcal{H}^{*} \mathbf{G}(H, F)$ of $H$ with coefficients in $F$ and with respect to $\mathbf{G}$ is the non-abelian cohomology of the diagram $\mathcal{C}^{\times} \mathbf{G}(H, F)$. In other words, we have

$$
\mathcal{H}^{0} \mathbf{G}(H, F)=\mathbb{H}^{0}\left(\mathcal{C}^{\times} \mathbf{G}(H, F)\right) \quad \text { and } \quad \mathcal{H}^{1} \mathbf{G}(H, F)=\mathbb{H}^{1}\left(\mathcal{C}^{\times} \mathbf{G}(H, F)\right) .
$$

We denote $\mathcal{Z}^{1} \mathbf{G}(H, F)$ the set $\mathbb{Z}^{1}\left(\mathcal{C}^{\times} \mathbf{G}(H, F)\right)$ and call it the set of Hopf 1-cocycles of $H$ with coefficients in $F$.

In particular, the non-abelian cohomology theory $\mathcal{H}^{*}(H, F)$ defined in [4] is the theory with respect to the identity functor.

We have now to add a technical lemma, which gives an alternative description of the 1-cocyle set $\mathcal{Z}^{1}(H, F)$. This result is the counterpart in the cohomology theory of Proposition 2.1 of [4] which was applied to the restricted cohomology theory studied in [3].

Lemma 2.0: For any $H$-comodule algebra, one has

$$
\mathcal{Z}^{1}(H, F)=\left\{X \in F \otimes H \quad \mid \quad\left(\operatorname{id}_{F} \otimes \varepsilon_{H}\right)(X)=1 \quad \text { and } \quad d^{2}(X) d^{0}(X)=d^{1}(X)\right\} .
$$

Proof: Pick an element $X \in F \otimes H$ satisfying the cocycle condition. We have to prove that the invertibility of $X$ is equivalent to the equality $\left(\operatorname{id}_{F} \otimes \varepsilon_{H}\right)(X)=1$. In the following, we set $x=$ $\left(\operatorname{id}_{F} \otimes \varepsilon_{H}\right)(X)$.

First suppose that $X$ is invertible in $F \otimes H$. Applying the map $\operatorname{id}_{F} \otimes \varepsilon_{H} \otimes \mathrm{id}_{H}$ to the cocycle relation, one gets the equality $(x \otimes 1) X=X$ in $F \otimes H$, hence $x \otimes 1=1 \otimes 1$. By use of $\operatorname{id}_{F} \otimes \varepsilon_{H}$, one deduces $x=1$.

Conversely, suppose that $x$ is equal to 1 . Set $Y=\left(\left(\operatorname{id}_{F} \otimes \mu_{H}\right) \circ\left(\varrho_{F} \otimes \sigma_{H}\right)\right)(X)$. Apply $\left(\operatorname{id}_{F} \otimes \mu_{H}\right) \circ\left(\operatorname{id}_{F} \otimes \operatorname{id}_{H} \otimes \sigma_{H}\right)$ to the cocycle condition $d^{2}(X) d^{0}(X)=d^{1}(X)$. The first picture of Figure 2.1 computes the left-hand side of the obtained equality whereas the second one calculates the right-hand side.

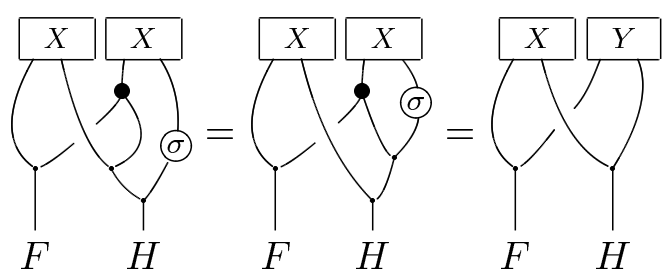

2.1.a

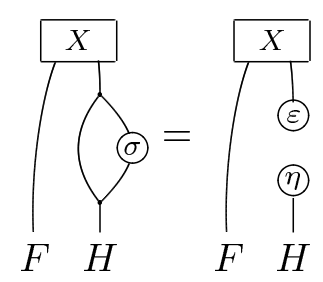

2.1.b

- Figure $2.1-$

So, one gets $X Y=x \otimes 1=1 \otimes 1$. In order to prove the left invertibility of $X$, apply the map $\left(\operatorname{id}_{F} \otimes \mu_{H}\right) \circ\left(\varrho_{F, H} \otimes \mu_{H}\right) \circ\left(\operatorname{id}_{F} \otimes \sigma_{H} \otimes \mathrm{id}_{H}\right)$ to the cocycle condition. This leads to the Hopf yoga of Figure 2.2. 


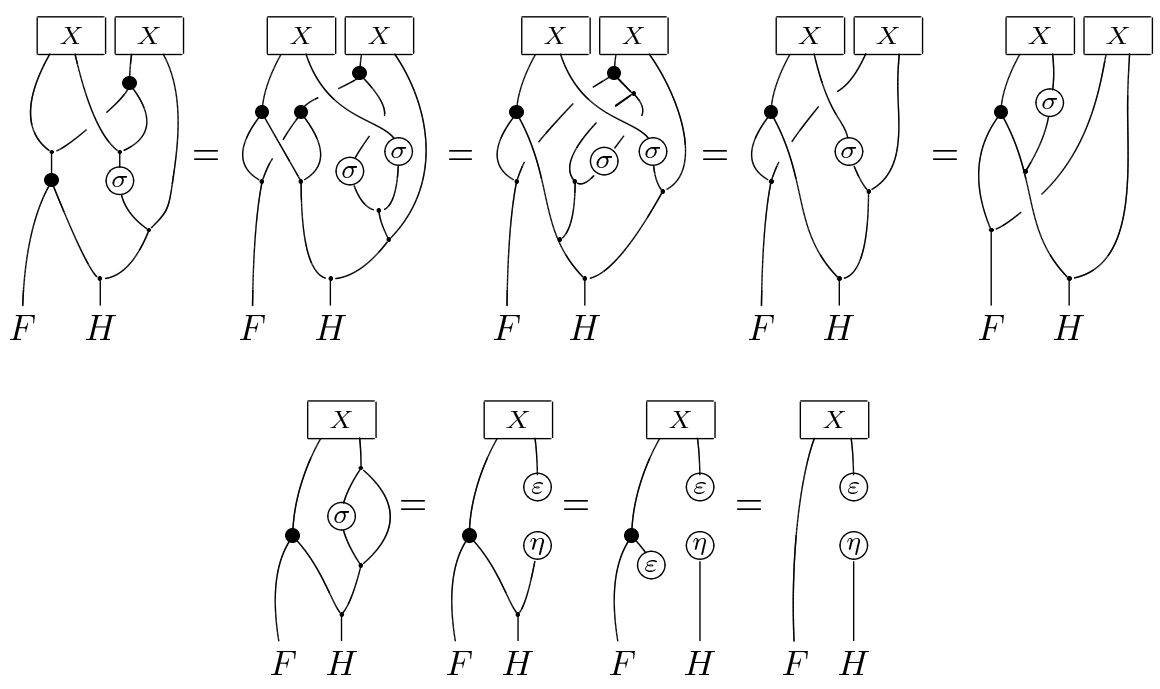

- Figure $2.2-$

This gives $Y X=x \otimes 1=1 \otimes 1$.

2.2. Cohomology theory with coefficients in Radford comodule algebras. Let $H$ be a Hopf algebra. Let $E$ be a Hopf algebra in the category $\mathscr{Y}_{H}^{H}$ and let $F$ be a Radford $E$-comodule algebra with $\varrho_{F, E}$ as $E$-comodule map. We paraphraze the above constructions in order to construct "braided" cohomology sets $\mathcal{H}_{\star}^{*}(E, F)$.

One checks that the maps $d^{*}$ defined by the formulae

$$
\begin{aligned}
& d^{0}(x)=\varrho_{F, E}(x), \quad d^{1}(x)=x \otimes 1, \\
& d^{0}(X)=\left(\varrho_{F, E} \otimes \operatorname{id}_{E}\right)(X), \quad d^{1}(X)=\left(\operatorname{id}_{F} \otimes \Delta_{E}\right)(X), \quad d^{2}(X)=X \otimes 1
\end{aligned}
$$

(where $x \in F$ and $X \in F \otimes E)$ induce the following pre-cosimplicial diagram $\mathcal{C}_{\star}(E, F)$ in the category of algebras:

$$
F \underset{d^{1}}{\stackrel{d^{0}}{\longrightarrow}} F \star E \underset{\frac{d^{0}}{\longrightarrow}}{\stackrel{\frac{d^{1}}{d^{2}}}{\longrightarrow}} F \star E \star E .
$$

For any endofunctor $\mathbf{G}$ of the category of algebras, one defines in the same way as before the non-abelian Hopf cohomology theory $\mathcal{H}_{\star}^{*} \mathbf{G}(E, F)$ with respect to $\mathbf{G}$ to be the non-abelian cohomology objects associated with the pre-cosimplicial diagram of groups $\mathcal{C}_{\star}^{\times} \mathbf{G}(E, F)$. The set of 1-cocycles are denoted by $\mathcal{Z}_{\star}^{1} \mathbf{G}(E, F)$.

The non-abelian Hopf cohomology objects $\mathcal{H}_{\star}^{*}(E, F)$ are by definition $\mathcal{H}_{\star}^{*} \mathbf{G}(E, F)$, with $\mathbf{G}$ being the identity functor. Notice that mutatis mutandis Lemma 2.0 is still valid for $\mathcal{Z}_{\star}^{1}(E, F)$. In the case when $H$ is equal to the ground ring $k$, then $E$ is a Hopf algebra and $F$ is an $E$-comodule algebra in the usual sense and one has the equality $\mathcal{H}_{\star}^{*}(E, F)=\mathcal{H}^{*}(E, F)$.

2.3. The decomposition Theorem. We are now able to state our main result, that is how to decompose, under the same hypotheses as before, the sets $\mathcal{H}^{*}(H \star E, F)$ in terms of $\mathcal{H}^{*}(H, F)$ and $\mathcal{H}_{\star}^{*}(E, F)$. The difficulty occures of course in the 1-cohomology level.

At this point we have to introduce the intermediate set $\mathcal{Z}^{1}(H, F){ }^{\star} \mathcal{Z}_{\star}^{1}(E, F)$ defined as follows. An element $\left[U^{H}, U^{E}\right]$ of $\mathcal{Z}^{1}(H, F) \stackrel{\star}{\times} \mathcal{Z}_{\star}^{1}(E, F)$ is a couple $\left(U^{H}, U^{E}\right)$ in $\mathcal{Z}^{1}(H, F) \times \mathcal{Z}_{\star}^{1}(E, F)$ such that $U^{H}$ and $U^{E}$ satisfy the compatibility relation drawn down in Figure 2.3. 


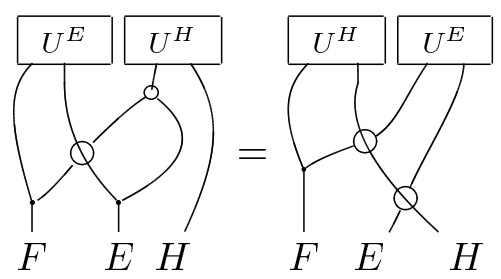

Compatibility relation

- Figure $2.3-$

Observe that $\mathcal{Z}^{1}(H, F) \stackrel{\star}{\times} \mathcal{Z}_{\star}^{1}(E, F)$ is a pointed set with $[1 \otimes 1,1 \otimes 1]$ as distinguished point.

Lemma 2.1: Let $H$ be a Hopf algebra. Let $E$ be a Hopf algebra in the category $\mathscr{Y} \mathscr{D}_{H}^{H}$ and let $F$ be a Radford E-comodule algebra. Then the group $F^{\times}$acts on the right on $\mathcal{Z}^{1}(H, F) \star \mathcal{Z}_{\star}^{1}(E, F)$ by

$$
\left[U^{H}, U^{E}\right]\left\llcorner x=\left[U^{H} \leftarrow x, U^{E}\llcorner x],\right.\right.
$$

where $\left[U^{H}, U^{E}\right]$ belongs to $\mathcal{Z}^{1}(H, F) \stackrel{\star}{\times} \mathcal{Z}_{\star}^{1}(E, F)$ and $x$ is an element of $F^{\times}$.

Proof: Take $\left[U^{H}, U^{E}\right]$ in $\mathcal{Z}^{1}(H, F) \stackrel{\star}{\times} \mathcal{Z}_{\star}^{1}(E, F)$ and $x$ in $F^{\times}$. One has to show that the elements $U^{H} \leftarrow x$ and $U^{E} \leftarrow x$ are compatible in the sense of Figure 2.3. By definition, $U^{H} \leftarrow x$ and $U^{E} \leftarrow x$ are respectively given by

$$
\begin{aligned}
& U^{H} \leftarrow x=\left(d^{1} x^{-1}\right) U^{H}\left(d^{0} x\right)=\left(x^{-1} \otimes 1\right) U^{H}\left(\varrho_{F, H}(x)\right) \text { and } \\
& U^{E} \leftarrow x=\left(d^{1} x^{-1}\right) U^{E}\left(d^{0} x\right)=\left(x^{-1} \otimes 1\right) U^{E}\left(\varrho_{F, E}(x)\right) .
\end{aligned}
$$

The product is the multiplication in $F \otimes H$ in the first line, and the multiplication in $F \star E$ in the second line. These formulae may be rewritten as shawn in Figure 2.4.

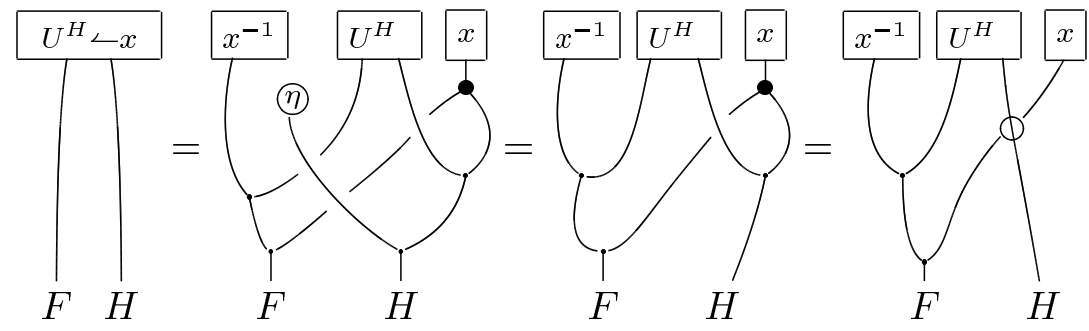

2.4.a

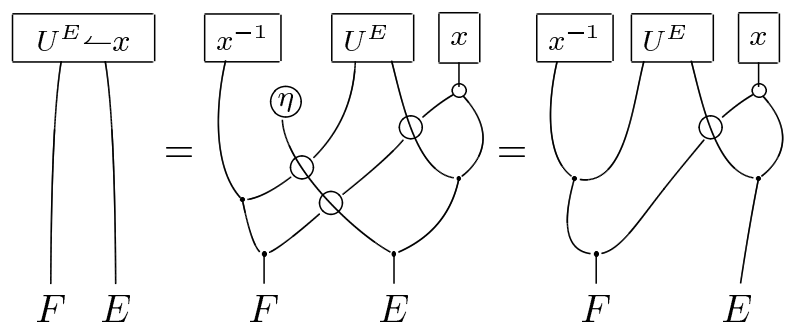

2.4.b

- Figure $2.4-$ 
Computing the left-hand side (respectively the right-hand side) of Relation 2.3 for the couple $\left(U^{H} \leftarrow x, U^{E} \leftarrow x\right)$, we obtain Figure 2.5 (respectively Figure 2.6)
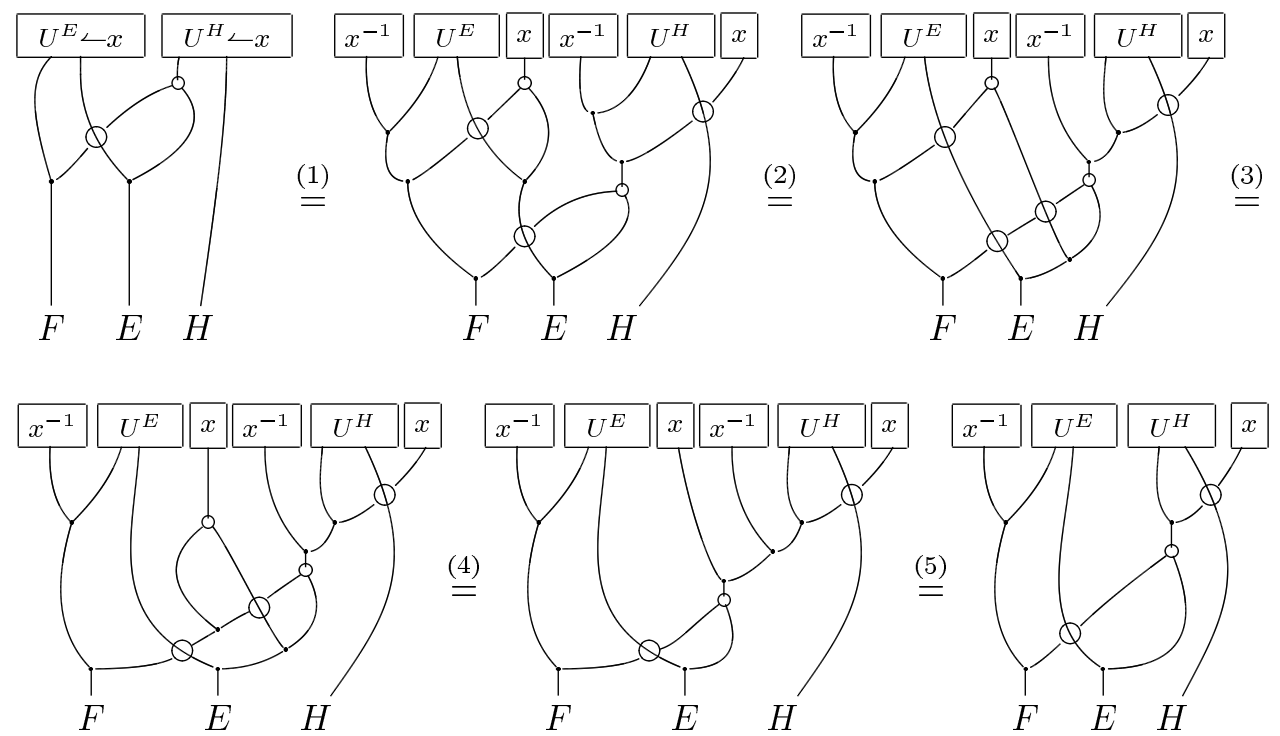

- Figure $2.5-$
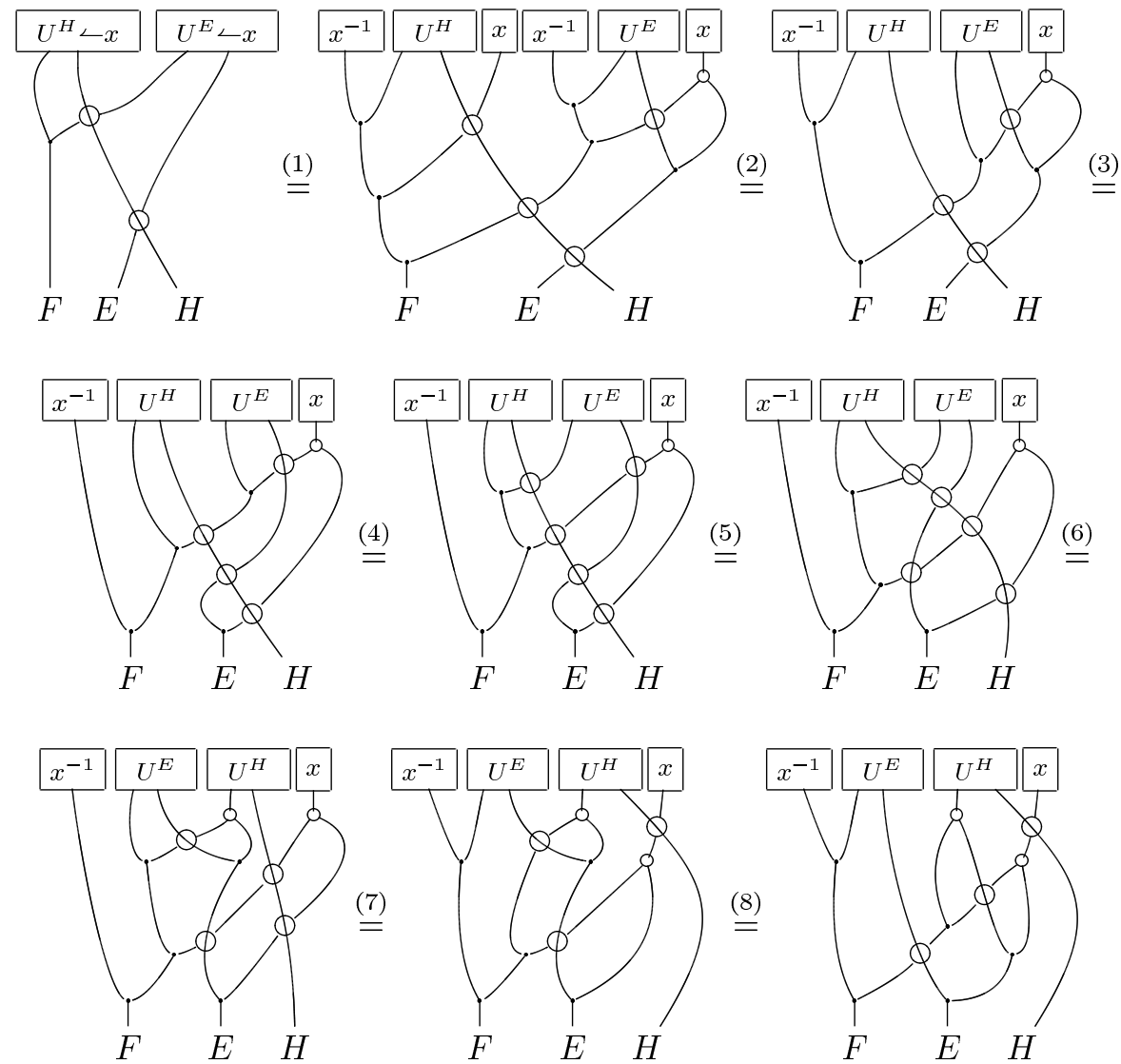

- Figure 2.6- 
Let us explain Figure 2.5. The first equality is a consequence of Figure 2.4. The second one comes from Relation 0.3.c applied to the $H$-module morphism $\mu_{E}$ and from the associativity of $\mu_{F}$ and of $\mu_{E}$. The third equality is implied by Relation 0.3.d applied to the $H$-comodule morphism $\mu_{F}$ and by the associativity of $\mu_{F}$. Equality (4) is deduced from Relation 0.8.a. Finally the fifth equality is the simplification of the product $x x^{-1}$, which appears by associativity of $\mu_{F}$.

We detail now Figure 2.6. The first equality is again a consequence of Figure 2.4. Equality (2) uses Relation 0.3.d for $\mu_{F}$, the associativity of $\mu_{F}$ and also the simplification $x x^{-1}=1$. The third equality comes from 0.3.d applied to the $H$-comodule morphism $\mu_{E}$ and from the associativity of $\mu_{F}$. Fourth equality is the sum of 0.3 .d for $\mu_{F}$ and the associativity of $\mu_{F}$. Equality (5) derives from Lemma 0.1 and from Relation 0.3.d. The sixth equality is the compatibility relation between $U^{H}$ and $U^{E}$ (Figure 2.3). Equality (7) translates the associativity of $\mu_{F}$ and 0.3 .d for $\varrho_{F, E}$. The eighth equality holds for the same reasons as the third one. Finally, by Relation 0.8.a, the two last pictures of Figure 2.5 and of Figure 2.6 are equal.

Theorem 2.2: Let $H$ be a Hopf algebra. Let $E$ be a Hopf algebra in the category $\mathscr{Y}_{H}^{H}$ and let $F$ be a Radford E-comodule algebra. Then one has the equality

$$
\mathcal{H}^{0}(H \star E, F)=\mathcal{H}^{0}(H, F) \cap \mathcal{H}_{\star}^{0}(E, F),
$$

and an isomorphism of pointed sets

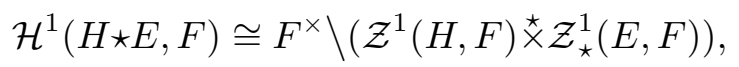

where the group $F^{\times}$acts on $\mathcal{Z}^{1}(H, F){ }^{\star} \mathcal{Z}_{\star}^{1}(E, F)$ by

$$
\left[U^{H}, U^{E}\right]\left\llcorner x=\left[U^{H} \leftarrow x, U^{E}\llcorner x] .\right.\right.
$$

Proof: 0-cohomology level. Recall that, after Proposition 1.1, the relationships between the three coaction maps on $F$ are given by

$$
\begin{aligned}
& \varrho_{F, H \star E}=\left(\varrho_{F, H} \otimes \mathrm{id}_{E}\right) \circ \varrho_{F, E}=\left(\operatorname{id}_{F} \otimes \tau_{E, H}\right) \circ\left(\varrho_{F, E} \otimes \mathrm{id}_{H}\right) \circ \varrho_{F, H} \\
& \varrho_{F, H}=\left(\operatorname{id}_{F} \otimes \operatorname{id}_{H} \otimes \varepsilon_{E}\right) \circ \varrho_{F, H \star E} \\
& \varrho_{F, E}=\left(\operatorname{id}_{F} \otimes \varepsilon_{H} \otimes \operatorname{id}_{E}\right) \circ \varrho_{F, H \star E}
\end{aligned}
$$

Let $x$ be an element of $\mathcal{H}^{0}(H \star E, F)$, that is an invertible element of $F$ such that $\varrho_{F, H \star E}(x)=$ $x \otimes 1 \otimes 1$. The equalities (2) and (3) applied to $x$ give $\varrho_{F, H}(x)=x \otimes 1$ and $\varrho_{F, E}(x)=x \otimes 1$. This means that $x$ belongs simultaneously to $\mathcal{H}^{0}(H, F)$ and to $\mathcal{H}_{\star}^{0}(E, F)$. Conversely, let $x$ be an element of $\mathcal{H}^{0}(H, F) \cap \mathcal{H}_{\star}^{0}(E, F)$. The first equality in (1) gives

$$
\varrho_{F, H \star E}(x)=\left(\left(\varrho_{F, H} \otimes \operatorname{id}_{E}\right) \circ \varrho_{F, E}\right)(x)=\left(\varrho_{F, H} \otimes \operatorname{id}_{E}\right)(x \otimes 1)=x \otimes 1 \otimes 1 .
$$

Hence $x$ is a 0 -cocycle of $H \star E$ with coefficients in $F$.

1-cocycle level. We first show that $\mathcal{Z}^{1}(H \star E, F)$ is isomorphic to $\mathcal{Z}^{1}(H, F) \stackrel{\star}{\times} \mathcal{Z}_{\star}^{1}(E, F)$. Let $X$ be an element of $\mathcal{Z}^{1}(H \star E, F)$. We define $X^{H} \in F \otimes H$ and $X^{E} \in F \otimes E$ by

$$
X^{H}=\left(\operatorname{id}_{F} \otimes \operatorname{id}_{H} \otimes \varepsilon_{E}\right)(X) \quad \text { and } \quad X^{E}=\left(\operatorname{id}_{F} \otimes \varepsilon_{H} \otimes \operatorname{id}_{E}\right)(X) .
$$

The element $X$ being invertible, so is $X^{H}$, $\operatorname{since~id~}_{H} \otimes \varepsilon_{E}$ is a morphism of algebras. The invertibility of $X^{E}$ is a consequence of Lemma 2.0. Indeed, one has $\left(\operatorname{id}_{F} \otimes \varepsilon_{E}\right)\left(X^{E}\right)=\left(\operatorname{id}_{F} \otimes \varepsilon_{H \star E}\right)(X)=1$.

By definition, $X$ satisfies the relation $d^{2}(X) d^{0}(X)=d^{1}(X)$, which pictorially looks like Relation 2.7.a, or after a slight simplification, like Relation 2.7.b. 


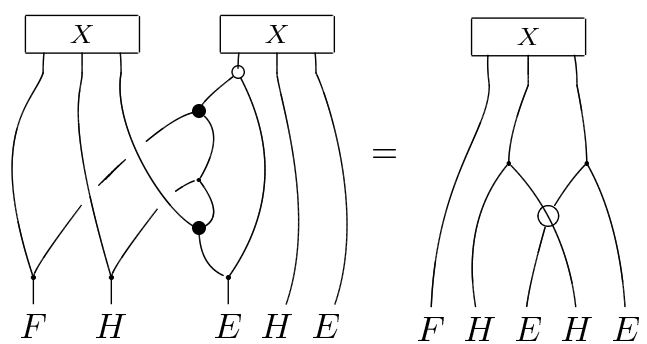

2.7.a

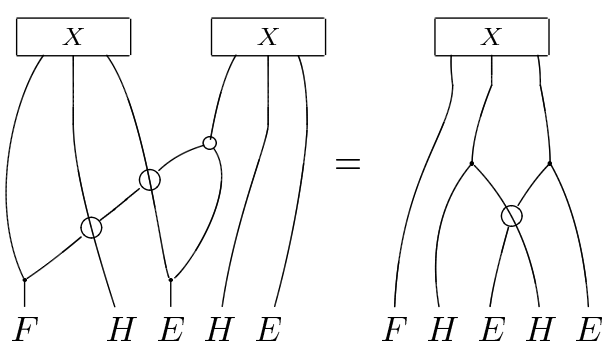

2.7.b

- Figure $2.7-$

The four manners to apply the counities to the $H$ - or $E$-outputs of 2.7.b. give rise to the four relations collected in Figure 2.8.

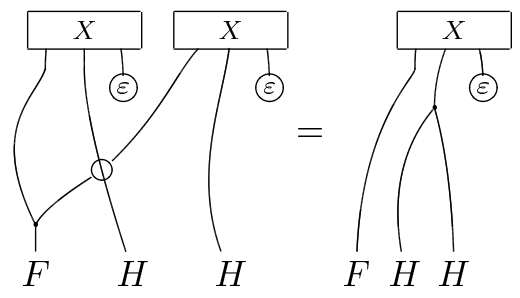

Relation 2.8.a

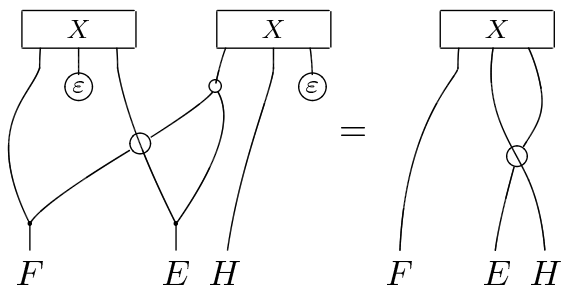

Relation 2.8.c

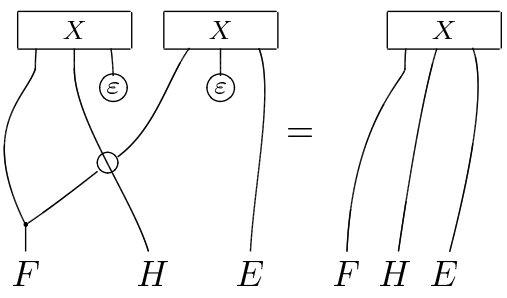

Relation 2.8.b

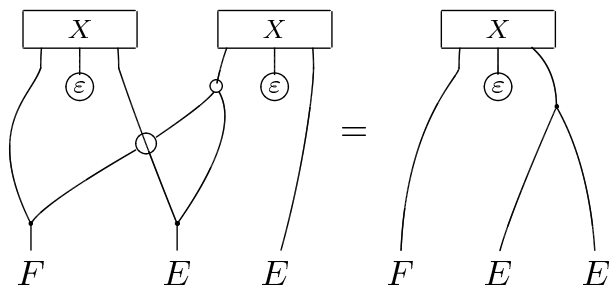

Relation 2.8.d

- Figure $2.8-$

Relation 2.8.a (respectively 2.8.d) says nothing else that $X^{H}$ (respectively $X^{E}$ ) belongs to $\mathcal{Z}^{1}(H, F)$ (respectively $\mathcal{Z}_{\star}^{1}(E, F)$ ). Relations 2.8.b and 2.8.c imply that $X^{H}$ and $X^{E}$ are compatible in the sense of Figure 2.3.

Conversely, pick $\left[X^{H}, X^{E}\right]$ in $\mathcal{Z}^{1}(H, F){ }^{\star} \mathcal{Z}_{\star}^{1}(E, F)$. Set

$$
X=\left(\left(\mu_{F} \otimes \mathrm{id}_{H} \otimes \mathrm{id}_{E}\right) \circ\left(\operatorname{id}_{F} \otimes \tau_{H, E} \otimes \mathrm{id}_{E}\right)\right)\left(X^{H} \otimes X^{E}\right),
$$

as depicted in Figure 2.9.

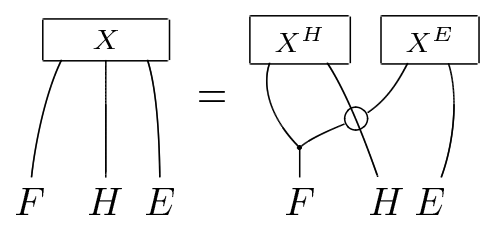

- Figure $2.9-$ 
Figure 2.10 below shows that $X$ verifies the cocycle condition. The first equality is a consequence of Relation 0.8.a. The second and the third one come from the associativity of the products $\mu_{H}$ and $\mu_{E}$. Equality (4) is implied by Relation 0.3.c applied to $\mu_{E}$ and by Relation 0.3.d applied to $\mu_{F}$. The fifth equality is the Compatibility relation 2.3. The next one is a consequence of Relation 0.3.d successively applied to $\varrho_{F, E}$, to $\tau_{E, F}$ and to $\mu_{E}$. The seventh equality is still a consequence of Relation 0.3.d applied to $\mu_{F}$. Equality (8) is the cocycle conditions for the two elements $X^{H}$ and $X^{E}$. Finally, the last one is Relation 0.3.c applied to $\Delta_{H}$.
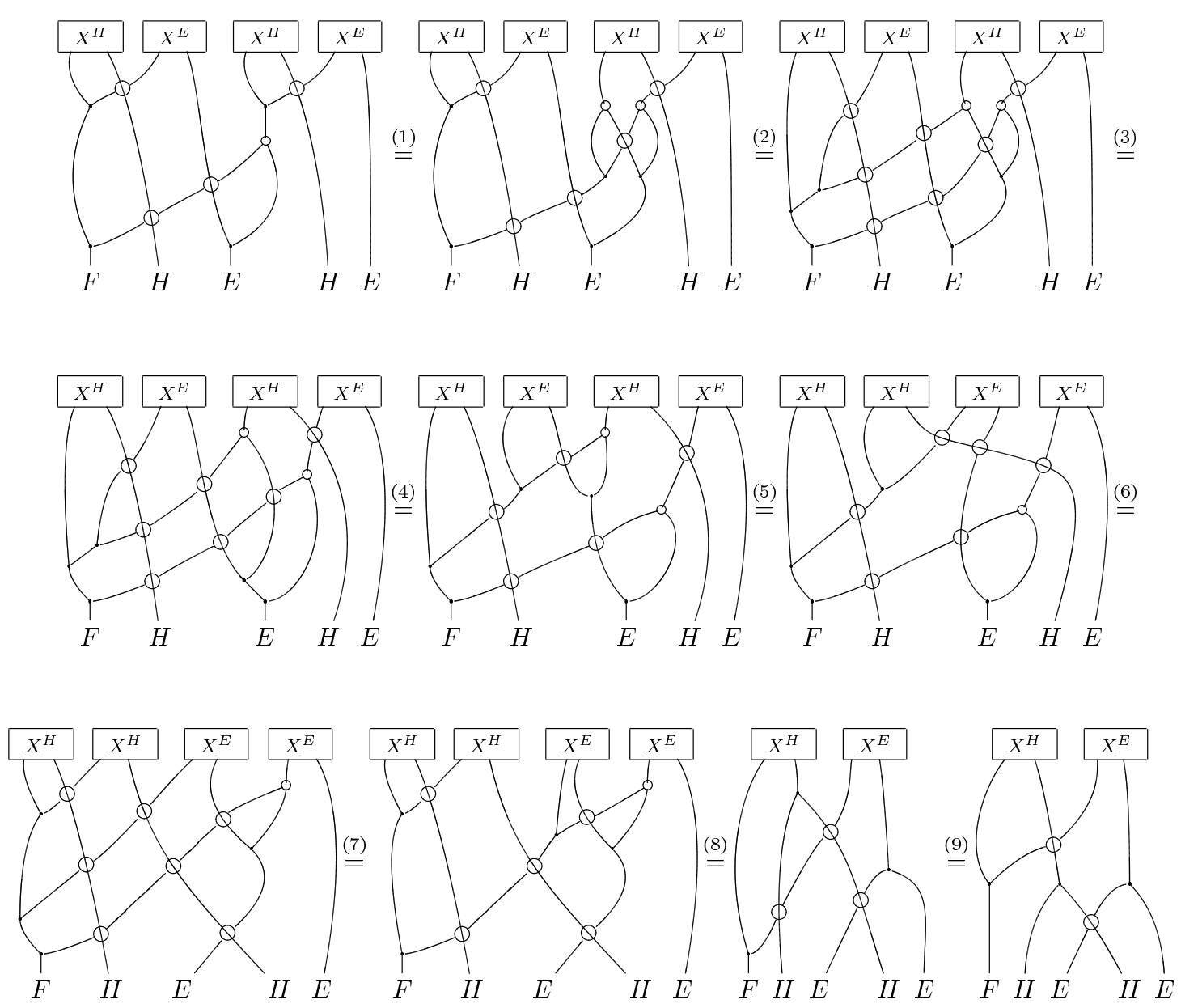

- Figure $2.10-$

In order to prove the invertibility of $X$, apply the map $\operatorname{id}_{F} \otimes \varepsilon_{H \star E}$ to $X$. By Relation 0.3.c and Lemma 2.0, the result is equal to 1 , hence, again by Lemma 2.0, $X$ belongs to $\mathcal{Z}^{1}(H \star E, F)$.

1-cohomology level. We leave to the Reader the proof of the two equalities

$$
(X \leftarrow x)^{H}=\left(X ^ { H } \llcorner x ) \text { and } \left(X\llcorner x)^{E}=\left(X^{E}\llcorner x)\right.\right.\right.
$$

where $X \in \mathcal{Z}^{1}(H \star E, F)$ and $x \in F^{\times}$. The first one is straightforward and the second one is a consequence of the Relations 0.4.c and 1.4.c. 
2.4. An exact sequence of 1-cohomology sets. In this paragraph, we construct under the previous hypotheses an exact sequence of pointed sets associated with the diagram

$$
H \stackrel{\operatorname{id}_{H} \otimes \eta_{E}}{\longrightarrow} H \star E \stackrel{\varepsilon_{H} \otimes \mathrm{id}_{E}}{\longrightarrow} E .
$$

To this end, we introduce the two following functors. First let $\mathbf{C}_{E}$ be the endofunctor of the category of $E$-comodule algebras defined on a $E$-comodule algebra $F$ by $\mathbf{C}_{E}(F)=F^{E}$. Here $F^{E}$ stands for the algebra of $E$-coinvariant elements of $F$. Secondly, let $\mathbf{T}_{H}$ be the endofunctor of the category of algebras given on an algebra $F$ by $\mathbf{T}_{H}(F)=F \otimes H$.

Notice that for any non-negative integer $i$, the module $F \otimes H^{\otimes i}$ can be endowed with the structure of $E$-comodule algebra through the formula $\varrho_{F \otimes H^{\otimes i}, E}(x \otimes \underline{h})=x_{0} \otimes \underline{h} \otimes x_{1}$. Here $\underline{h}$ belongs to $H^{\otimes i}$, the element $x$ belongs to $F$, and we set $\varrho_{F, E}(x)=x_{0} \otimes x_{1}$. With respect to this $E$-comodule structure, $\mathcal{C}(H, F)$ may easily be seen to be a pre-cosimplicial diagram in the category of $E$-comodule algebras. One is therefore allowed to form the cohomology sets $\mathcal{H}^{*} \mathbf{C}_{E}(H, F)$.

Lemma 2.3: Let $H$ be a Hopf algebra. Let $E$ be a Hopf algebra in the category $\mathscr{Y} \mathscr{D}_{H}^{H}$ and let $F$ be a Radford E-comodule algebra. When $H$ is flat as a $k$-module, there is an isomorphism

$$
\mathcal{H}^{*} \mathbf{C}_{E}(H, F) \cong \mathcal{H}^{*}\left(H, F^{E}\right) .
$$

Proof: By flatness, $\left(F \otimes H^{\otimes i}\right)^{E}$ is isomorphic to $F^{E} \otimes H^{\otimes i}$, for $i=0,1,2$. This implies an isomorphism $\mathcal{C}^{*} \mathbf{C}_{E}(H, F) \cong \mathcal{C}^{*}\left(H, F^{E}\right)$ on the co-simplicial level, hence on the cohomology sets.

As explained in 1.3, the algebras $F, F \star E$ and $F \star E \star E$ are $H$-comodule algebras. Consider the two morphisms of cosimplicial algebras $\mathcal{C}_{\star}(E, F) \stackrel{\eta}{\stackrel{\varrho}{\rightleftarrows}} \mathcal{C}_{\star} \mathbf{T}_{H}(E, F)$ summarised in the following diagram:

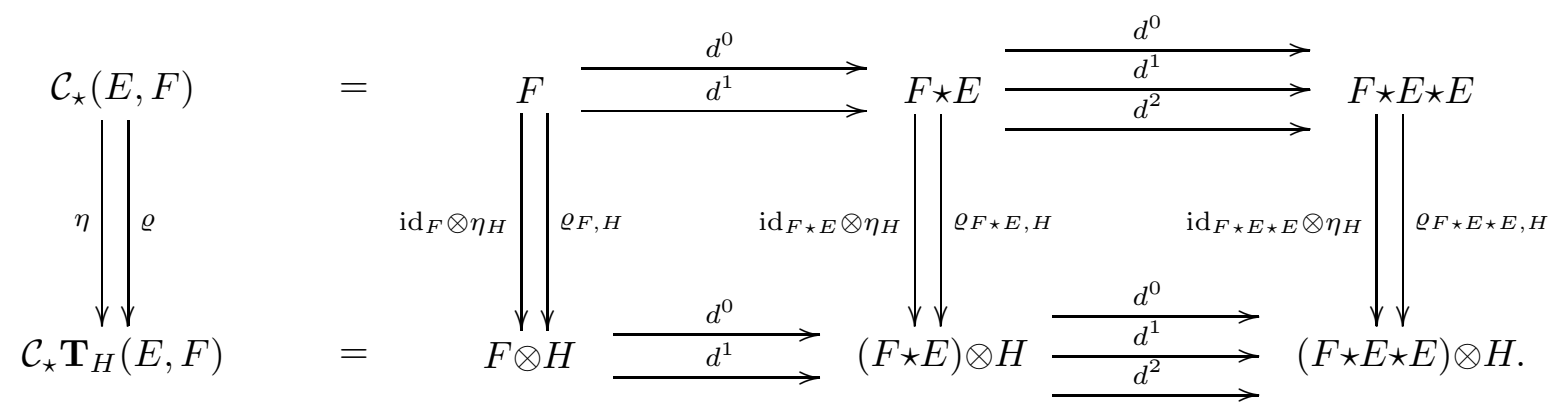

They give rise to the two morphisms $\mathcal{H}_{\star}^{*}(E, F) \stackrel{\eta}{\underset{\varrho}{\longrightarrow}} \mathcal{H}_{\star}^{*} \mathbf{T}_{H}(E, F)$ on the cohomology level. Define $\mathcal{H}_{\star}^{*}(E, F)^{H}$ to be the equalizer of these two arrows.

Theorem 2.4: Let $H$ be a Hopf algebra. Let $E$ be a Hopf algebra in the category $\mathscr{Y}_{H}^{H}$ and let $F$ be a Radford E-comodule algebra. The two maps $\iota: F \otimes H \longrightarrow(F \otimes H) \times(F \otimes E)$ and $\pi:(F \otimes H) \times(F \otimes E) \longrightarrow(F \otimes E)$ defined by

$$
\iota(X)=(X, 1) \quad \text { and } \quad \pi(X, Y)=Y
$$

give rise to the following exact sequence of pointed sets, in which $\iota$ is injective:

$$
\mathcal{H}^{1} \mathbf{C}_{E}(H, F) \stackrel{\iota}{\longrightarrow} \mathcal{H}^{1}(H \star E, F) \stackrel{\pi}{\longrightarrow} \mathcal{H}_{\star}^{1}(E, F)^{H} .
$$


Proof: We first show that $\iota$ induces an injective map, still denoted by $\iota$, from $\mathcal{H}^{1} \mathbf{C}_{E}(H, F)$ to $F^{\times} \backslash\left(\mathcal{Z}^{1}(H, F){ }_{\star}^{\star} \mathcal{Z}_{\star}^{1}(E, F)\right)$, which is isomorphic to $\mathcal{H}^{1}(H \star E, F)$ by Theorem 2.2. To this end take $X=x \otimes h$ an element of $\mathcal{Z}^{1} \mathbf{C}_{E}(H, F)$. Notice that the $E$-coinvariance of $X$ is equivalent to the relation $\varrho_{F, E}(x) \otimes h=x \otimes 1 \otimes h$, which is itself equivalent to the Compatibility relation 2.3 between $X$ and $1 \otimes 1$. So $[X, 1]$ belongs to $\mathcal{Z}^{1}(H, F){ }^{\star} \mathcal{Z}_{\star}^{1}(E, F)$.

Let $X$ and $X^{\prime}$ be two elements of $\mathcal{Z}^{1} \mathbf{C}_{E}(H, F)$ such that $X^{\prime}=X<x$ with $x \in\left(F^{E}\right)^{\times}$. One has $[X, 1]<x=[X \leftarrow x, 1<x]$. But since $x$ is $E$-coinvariant, one also has $1 \leftarrow x=$ $\left(x^{-1} \otimes 1\right) \varrho_{F, E}(x)=1$. As a consequence, $\iota$ induces a map on the quotients.

Suppose now that $X$ and $X^{\prime}$ have the same image by $\iota$. This means that there exists an element $x \in F^{\times}$such that $[X, 1]<x=\left[X^{\prime}, 1\right]$, which is equivalent to the two equalities $X<x=X^{\prime}$ and $1=1 \leftarrow x$. The latter equality reflects that $x$ belongs to the group $\left(F^{E}\right)^{\times}$. Therefore $X$ and $X^{\prime}$ are cohomologous in $\mathcal{Z}^{1} \mathbf{C}_{E}(H, F)$. Hence $\iota$ is injective.

We show that the image of $\pi$ lies in $\mathcal{H}_{\star}^{1}(E, F)^{H}$. To this end, let $[X, Y] \in \mathcal{Z}^{1}(H, F){ }^{\star} \mathcal{Z}_{\star}^{1}(E, F)$ represent an element of $\mathcal{H}^{1}(H \star E, F)$. Figure 2.11 proves that "multiplying" the right-hand side of the Compatibility relation 2.3 between $X$ and $Y$ by $X^{-1}$ leads to $\varrho_{F \star E, H}(Y)$. Indeed, the first equality is a consequence of the associativity of the algebra $F$. The second one comes from Relation 0.4.a, whereas the third one reflects $X^{-1} X=1$. Finally, the last equality is given by Relation 0.4.d.

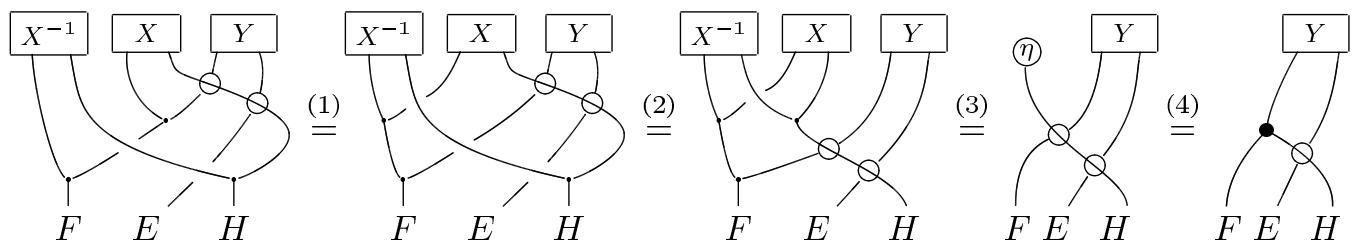

- Figure $2.11-$

In Figure 2.12 the left-hand side of the Compatibility relation 2.3 between $X$ and $Y$ is "multiplied" by $X^{-1}$ in the same manner as above. By inserting $\eta_{E}$ and $\eta_{H}$, one obtains the element $(Y \otimes 1)<X$ in $(F \star E) \otimes H$.

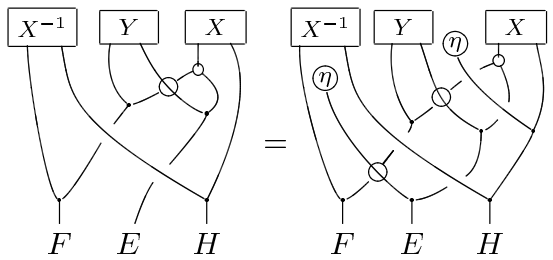

- Figure $2.12-$

So the elements $\varrho_{F \star E, H}(Y)$ and $Y \otimes 1$ are cohomologous in $\mathcal{Z}_{\star}^{1} \mathbf{T}_{H}(E, F)$, and therefore $\pi([X, Y])$ belongs to the set $\mathcal{H}_{\star}^{1}(E, F)^{H}$.

It is obvious that the image of $\iota$ is included in the preimage of 1 by $\pi$. Conversely, take $[X, Y]$ in $\mathcal{Z}^{1}(H, F) \stackrel{\star}{\times} \mathcal{Z}_{\star}^{1}(E, F)$ such that its class in $\mathcal{H}^{1}(H \star E, F)$ has a trivial image via $\pi$. This means that there exists an element $x$ in $F^{\times}$such that $Y=1<x$. As $[X, 1<x]$ is equal to $\left[X<x^{-1}, 1\right]<x$, the class of $[X, Y]$ is also represented by $\left[X<x^{-1}, 1\right]$. By the remark formulated above, this 
means that $X<x^{-1}$ is $E$-coinvariant. In other words, the class of $[X, Y]$ belongs to the image of $\iota$.

\section{Computation of examples.}

3.1. When $F=E$.

Proposition 3.1: Let $H$ be a Hopf algebra and $E$ be a Hopf algebra in the category $\mathscr{Y} \mathscr{D}_{H}^{H}$. Then one has the equality

$$
\mathcal{H}^{0}(H \star E, E)=k^{\times}
$$

and an isomorphism of pointed sets

$$
\mathcal{H}^{1}(H \star E, E) \cong \operatorname{Gr}(H)
$$

Here $\operatorname{Gr}(H)$ stands for the set of grouplike elements of $H$.

Proof:

0-cohomology level. By definition, one immediately obtains that $\mathcal{H}_{\star}^{0}(E, E)$ is equal to $k^{\times}$. Since the group $k^{\times}$is included in $\mathcal{H}^{0}(H, E)$, Theorem 2.2 implies the first assertion.

1-cohomology level. We use the exact sequence given in Theorem 2.4. The computations of Example 1.2.3 in [4] can be adapted here, so the 1-cohomology set $\mathcal{H}_{\star}^{1}(E, E)$ is equal to $\{1\}$. Therefore $\mathcal{H}_{\star}^{1}(E, E)^{H}$ is trivial. On the other hand, let $x \otimes h$ be an element of $\mathcal{Z}^{1} \mathbf{C}_{E}(H, E)$. The $E$-coinvariance of $x \otimes h$ is expressed by the equality $x_{1} \otimes h \otimes x_{2}=x \otimes h \otimes 1$. Apply $\varepsilon_{E}$ to the first component of the tensor product in order to get $x \otimes h=1 \otimes \varepsilon_{E}(x) h$. Call $1 \otimes h^{\prime}$ the latter element. The cocycle condition fulfilled by $1 \otimes h^{\prime}$ implies that $h^{\prime}$ belongs to $\operatorname{Gr}(H)$. Conversely, starting with $h^{\prime} \in \operatorname{Gr}(H)$, one checks that the element $1 \otimes h^{\prime}$ is in $\mathcal{Z}^{1} \mathbf{C}_{E}(H, E)$. Moreover $E^{E}$ is equal to the ground ring $k$, hence $\mathcal{H}^{1} \mathbf{C}_{E}(H, E)$ is isomorphic to $\operatorname{Gr}(H)$. The exact sequence of Theorem 2.4 becomes $\operatorname{Gr}(H) \stackrel{\iota}{\longrightarrow} \mathcal{H}^{1}(H \star E, F) \longrightarrow 1$. The injectivity of $\iota$ allows us to conclude.

In the case when $E$ is equal to the ground ring $k$, one recovers the computation of $\mathcal{H}^{*}(H, k)$ performed in Example 1.2.2 of [4].

3.2. Taft algebras. Let here $k$ be a field. Fix $n$ an integer with $n \geq 2$ and $\zeta$ a primitive $n$-th root of unity in $k$. Consider the Taft algebra $H_{n^{2}}$ generated by two elements $g$ and $h$ submitted to the relations $g^{n}=1, h^{n}=0$ and $h g=\zeta g h([10])$. On the generators the comultiplication, the antipode, and the counit of $H_{n^{2}}$ are given by

$$
\begin{array}{ll}
\Delta(g)=g \otimes g, & \Delta(h)=h \otimes g+1 \otimes h, \\
\sigma(g)=g^{n-1}, & \sigma(h)=-\zeta^{-1} g^{n-1} h, \\
\varepsilon(g)=1, & \varepsilon(h)=0 .
\end{array}
$$

Denote by $E_{n}$ the subalgebra of $H_{n^{2}}$ generated by $h$. Via $\Delta_{H_{n^{2}}}$, the algebra $E_{n}$ is naturally endowed with a structure of $H_{n^{2}}$-comodule algebra.

The Taft algebra $H_{n^{2}}$ is in fact a Radford product. Indeed, let $G$ be the cyclic group of order $n$ generated by $u$. As usually, denote by $k[G]$ the Hopf algebra of the group $G$. Notice that the co-opposite algebra of $k[G]$ is a Hopf algebra, since $k[G]$ is cocommutative. Let $E$ be the algebra generated by $y$ submitted to the relation $y^{n}=0$. Endow $E$ with the $k[G]$-module and $k[G]$-comodule structures defined for any nonnegative integers $i$ and $j$ by:

$$
y^{i} \cdot u^{j}=\zeta^{i j} y^{i} \quad \text { and } \quad \varrho_{E, k[G]}\left(y^{i}\right)=y^{i} \otimes u^{i} .
$$


Then $E$ is an object of $\mathscr{Y}_{\mathscr{D}_{[}^{k[G]}}^{k[G]}$. Equip $E$ with the maps $\Delta_{E}, \varepsilon_{E}$, and $\sigma_{E}$ given on $y^{i} \in E$ by

$$
\Delta_{E}\left(y^{i}\right)=\sum_{s=0}^{i}\left(\begin{array}{l}
i \\
s
\end{array}\right)_{\zeta} y^{s} \otimes y^{i-s}, \quad \varepsilon_{E}\left(y^{i}\right)=0, \text { when } i>0, \quad \text { and } \quad \sigma_{E}\left(y^{i}\right)=(-1)^{i} \zeta^{\frac{i(i-1)}{2}} y^{i} .
$$

The symbol $\left(\begin{array}{l}i \\ s\end{array}\right)_{\zeta}$ denotes the $\zeta$-analogue of the binomial coefficient. Provided with all these data, $E$ becomes a Hopf algebra in the braided category $\mathscr{Y}_{\mathscr{D}_{k[G]}^{k[G]}}$.

One may now form the Radford product $k[G] \star E$, and convince oneself that it is isomorphic to the Taft algebra $H_{n^{2}}$ by sending $1 \star y$ to $h$ and $u \star 1$ to $g$. In this correspondence $E$ is isomorphic to $E_{n}$ as an algebra.

As an immediate consequence of Proposition 3.1, we obtain the following result, which proves the conjecture formulated in [4]. It generalizes Proposition 1.6 of [4].

Corollary 3.2: There is an equality of groups

$$
\mathcal{H}^{0}\left(H_{n^{2}}, E_{n}\right)=k^{\times}
$$

and an isomorphism of pointed sets

$$
\mathcal{H}^{1}\left(H_{n^{2}}, E_{n}\right) \cong\left\{1 \otimes g^{k} \mid 0 \leq k \leq n-1\right\} .
$$

The class of Hopf algebras of the type $k[G] \star E$ is very large. We refer to [1] for general results and various examples, as the quantum plane or the Lie colour algebras. By Proposition 3.1, one always gets the equality $\mathcal{H}^{0}(k[G] \star E, E)=k^{\times}$and the isomorphism $\mathcal{H}^{*}(k[G] \star E, E) \cong G$.

3.3. Dual of group algebras. Let $D$ be a finite group with neutral element 1 . Denote by $k^{D}$ the $k$-free Hopf algebra over the $k$-basis $\left\{\delta_{d}\right\}_{g \in D}$, with the following structure maps: the multiplication is given by $\delta_{d} \cdot \delta_{d^{\prime}}=\partial_{d, d^{\prime}} \delta_{d}$, where $\partial_{d, d^{\prime}}$ stands for the Kronecker symbol of $d$ and $d^{\prime}$; the unit in $k^{D}$ is the element $1=\sum_{d \in G} \delta_{d}$; the comultiplication $\Delta_{k^{D}}$ is defined by $\Delta_{k^{D}}\left(\delta_{d}\right)=\sum_{u v=d} \delta_{u} \otimes \delta_{v}$; the counit $\varepsilon_{k^{D}}$ is defined by $\varepsilon_{k^{D}}\left(\delta_{d}\right)=\partial_{d, 1} 1$; the antipode $\sigma_{k^{D}}$ sends $\delta_{d}$ on $\delta_{d^{-1}}$. When $k$ is a field, then $k^{D}$ is the dual of the usual group algebra $k[D]$.

In [4], we related the non-abelian Hopf cohomology theory of $k^{D}$ with the non-abelian cohomology theory of the group $D$ (Theorem 1.5). Recall briefly the definitions of the non-abelian cohomology theory $\mathrm{H}^{i}(D, C)$ (with $i=0,1$ ) of a group $D$ with coefficients in a $D$-group $C$. The 0-cohomology object $\mathrm{H}^{0}(D, C)$ is the group $C^{D}$ of invariant elements of $C$ under the action of $D$. The set $\mathrm{Z}^{1}(D, C)$ of 1-cocycles is given by

$$
\mathrm{Z}^{1}(D, C)=\left\{\beta: D \longrightarrow C \mid \beta\left(d d^{\prime}\right)=\beta(d)^{d}\left(\beta\left(d^{\prime}\right)\right), \quad \forall d, d^{\prime} \in D\right\} .
$$

The group $C$ acts on the right on $\mathrm{Z}^{1}(D, C)$ by

$$
(\beta \leftarrow x)(d)=x^{-1} \beta(d)^{d} x,
$$

where $x \in C, \beta \in \mathrm{Z}^{1}(D, C)$, and $d \in G$.

The non-abelian 1-cohomology set $\mathrm{H}^{1}(D, C)$ is the left quotient $C \backslash \mathrm{Z}^{1}(D, C)$. It is pointed with distinguished point the class of the constant map 1:D $\longrightarrow$. Notice that the non-abelian cohomology theory of groups may also be interpreted as the non-abelian cohomology theory associated to a pre-cosimplicial diagram of groups [4]. 
Let $F$ be a $k^{D}$-comodule algebra. Recall [4] that, for any $x \in F$, the formula

$$
\varrho_{E, k^{D}}(x)=\sum_{d \in D}{ }^{d} x \otimes \delta_{d}
$$

defines a left action of the group $D$ on the algebra $F$, hence on the group $F^{\times}$. Theorem 1.5 of [4] states an isomorphism between $\mathcal{H}^{*}\left(k^{D}, F\right)$ and $\mathrm{H}^{*}\left(D, F^{\times}\right)$

Consider now the case where $D$ is a semi-direct product of groups. Let $G$ and $A$ be two finite groups such that $G$ acts on the left on $A$. In our conventions the semi-direct group $G \rtimes A$ is the set $G \times A$ endowed with the product given for $(g, a),(h, b) \in G \times A$ by

$$
(g, a)(h, b)=\left(g h,^{h^{-1}} a b\right) .
$$

The co-opposite algebra of $k^{G}$ is still a Hopf algebra, so we can consider the braided monoidal category $\mathscr{Y}_{k^{G}}^{k^{G}}$. Equip $k^{A}$ with the $k^{G}$-module and $k^{G}$-comodule structures given by

$$
\delta_{a} \cdot \delta_{g}=\partial_{g, 1} \delta_{a} \quad \text { and } \quad \varrho_{k^{A}, k}\left(\delta_{a}\right)=\sum_{h \in G} \delta_{h_{a} \otimes \delta_{h}}
$$

for any $a \in A$ and $g \in G$. Then $k^{A}$ becomes a Hopf algebra in the Yetter-Drinfeld category $\mathscr{Y}_{k^{G}}^{k^{G}}$ and one has an isomorphism of Hopf algebras

$$
k^{G} \star k^{A} \cong k^{G \rtimes A}
$$

in which the element $\delta_{g} \star \delta_{a}$ corresponds with $\delta_{(g, a)}$. When the action of $G$ on $A$ is trivial, then $k^{A}$ is equipped with the trivial $k^{G}$-module and $k^{G}$-comodule structures and the above isomorphism reduces to

$$
k^{G} \otimes k^{A} \cong k^{G \times A}
$$

Theorem 1.5 of [4] and the previous Theorem 2.2 imply the following result:

Proposition 3.3: Let $G$ and $A$ be two finite groups such that $G$ acts on the left on $A$. Let $F$ be a $k^{G \rtimes A}$-comodule algebra. There is an equality of groups

$$
\mathrm{H}^{0}\left(G \rtimes A, F^{\times}\right)=\mathrm{H}^{0}\left(G, F^{\times}\right) \cap \mathrm{H}^{0}\left(A, F^{\times}\right),
$$

and an isomorphism of pointed sets

$$
\mathrm{H}^{1}\left(G \rtimes A, F^{\times}\right) \cong F^{\times} \backslash\left(\mathcal{Z}^{1}\left(k^{G}, F\right) \stackrel{\star}{\times} \mathcal{Z}_{\star}^{1}\left(k^{A}, F\right)\right) .
$$




\section{Digression: The non-abelian cohomology theory for semi-direct products of groups.} With the help of the Hopf algebra machinery, we are able by Proposition 3.3 to compute the nonabelian cohomology theory of a semi-direct group with coefficients in a group of a particular type: the group of invertible elements of a certain algebra. One should now like to provide such a calculation in the case of an arbitrary coefficient group. In the present section, we show how to to do this directly, using only the basic definitions of the non-abelian cohomology theory ([6], [7]). The results we obtain are the same as those in Proposition 3.3. This closeness may be explained by a metamathematical fantasy suggested by several mathematicians, the "field of one element" $\mathbf{F}_{1}$ (see [8]). Indeed, in this philosophy, a group is nothing but a Hopf algebra over the field $\mathbf{F}_{1}$.

Let now $G$ and $A$ be two finite groups such that $G$ acts on the left on $A$. Let $C$ be a group. It is easy to see that $C$ is a $G \rtimes A$-group if and only if $C$ is both a $G$-group and an $A$-group such that the two actions satisfy the following compatibility law:

$$
{ }^{g}\left({ }^{a} x\right)={ }^{g_{a}}\left({ }^{g} x\right)
$$

for any $g \in G, a \in A$, and $x \in C$. This is to say that the $G$-group $A$ acts on the left on $C$ or, in other words, that the canonical map $A \times C \longrightarrow C$ is a morphism of $G$-sets. The dictionary between the several actions is given by ${ }^{(g, a)}(x)={ }^{g}\left({ }^{a} x\right)$, for $(g, a) \in G \rtimes A$ and $x \in C$.

We define now the set $\mathrm{Z}^{1}(G, C) \stackrel{\star}{\times} \mathrm{Z}^{1}(A, C)$. It elements $[\gamma, \alpha]$ are couples in $\mathrm{Z}^{1}(G, C) \times \mathrm{Z}^{1}(A, C)$ such that $\gamma$ and $\alpha$ satisfy the compatibility relation

$$
\gamma(g){ }^{g} \alpha(a)=\alpha\left({ }^{g} a\right){ }^{g} a \gamma(g) .
$$

Observe that $\mathrm{Z}^{1}(G, C) \stackrel{\star}{\times} \mathrm{Z}^{1}(A, C)$ is a pointed set with $[1,1]$ as distinguished point. The Reader easily checks that the group $C$ acts on the right on $\mathrm{Z}^{1}(G, C) \stackrel{\star}{\times} \mathrm{Z}^{1}(A, C)$ by

$$
[\gamma, \alpha]<c=[\gamma<c, \alpha<c]
$$

for $[\gamma, \alpha] \in \mathrm{Z}^{1}(G, C) \stackrel{\star}{\times} \mathrm{Z}^{1}(A, C)$ and $c \in C$.

The group counterpart of Theorem 2.2 is the following result:

Proposition 4.1: Let $G$ and $A$ be two finite groups such that $G$ acts on the left on $A$. Let $C$ be a $G \rtimes A$-group. There is an equality of groups

$$
\mathrm{H}^{0}(G \rtimes A, C)=\mathrm{H}^{0}(G, C) \cap \mathrm{H}^{0}(A, C),
$$

and an isomorphism of pointed sets

$$
\mathrm{H}^{1}(G \rtimes A, C) \cong C \backslash\left(\mathrm{Z}^{1}(G, C) \stackrel{\star}{\times} \mathrm{Z}^{1}(A, C)\right) .
$$

Proof: The proof in the 0-level is a direct consequence of the definitions. On the first level, it is enough to exhibit an explicit isomorphism between $\mathrm{Z}^{1}(G \rtimes A, C)$ and $\mathrm{Z}^{1}(G, C) \stackrel{\star}{\times} \mathrm{Z}^{1}(A, C)$. The correspondence $\beta \longmapsto[\gamma, \alpha]$ defined by $\gamma(g)=\beta(g, 1)$ and $\alpha(a)=\beta(1, a)$, where $g \in G$ and $a \in A$, realize this isomorphism. Conversely, a compatible couple $[\gamma, \alpha]$ gives raise to the element $\beta \in \mathrm{Z}^{1}(G \rtimes A, C)$ defined by $\beta(g, a)=\gamma(g){ }^{g} \alpha(a)$, for any $(g, a) \in G \rtimes A$. 
Notice that $k^{C}$ is naturally equipped with a $k^{G} \star k^{A}$-comodule algebra structure when $C$ is a $G \rtimes A$-group. Via Proposition 3.3 and [4, Theorem 1.5], one obtains an equality of groups

$$
\mathrm{H}^{0}\left(G \rtimes A,\left(k^{C}\right)^{\times}\right)=\mathrm{H}^{0}\left(G,\left(k^{C}\right)^{\times}\right) \cap \mathrm{H}^{0}\left(A,\left(k^{C}\right)^{\times}\right),
$$

and an isomorphism of pointed sets

$$
\mathrm{H}^{1}\left(G \rtimes A,\left(k^{C}\right)^{\times}\right) \cong\left(k^{C}\right)^{\times} \backslash\left(\mathrm{Z}^{1}\left(G,\left(k^{C}\right)^{\times}\right) \stackrel{\star}{\times} \mathrm{Z}^{1}\left(A,\left(k^{C}\right)^{\times}\right)\right) .
$$

Here we could replace $\mathcal{Z}_{\star}^{1}\left(k^{A}, k^{C}\right)$ by $\mathcal{Z}^{1}\left(k^{A}, k^{C}\right)$, using the fact that the braiding in $\mathscr{Y}_{k^{G}}^{k^{G}}$ is the usual flip for the objects of type $k^{D}$, for any $G$-group $D$. This latter result differs from that stated in Proposition 4.1, since the group of invertible elements of $k^{C}$ is in general not isomorphic to $C$ (unless $k=\mathbf{F}_{1}$ !).

We end this digression by observing that the group counterpart of Theorem 2.4 is the exact sequence of pointed sets

$$
\mathrm{H}^{1}\left(G, C^{A}\right) \stackrel{\iota}{\longrightarrow} \mathrm{H}^{1}(G \rtimes A, C) \stackrel{\pi}{\longrightarrow} \mathrm{H}^{1}(A, C)^{G}
$$

(with $\iota$ injective). Through the isomorphism described in Proposition 4.1, the maps $\iota$ and $\pi$ may be simply expressed by the formulae $\iota(\gamma)=[\gamma, 1]$ and $\pi([\gamma, \alpha])=\alpha$. This cohomological sequence is by Serre's theory [7] recovered as the one associated with the exact sequence of groups $1 \longrightarrow A \longrightarrow G \rtimes A \longrightarrow G \longrightarrow 1$.

\section{REFERENCES}

[1] D. Fischman, S. Montgomery, A Schur double centralizer theorem for cotriangular Hopf algebras and generalized Lie algebras, J. Algebra 168, (1994), nº 2, $594-614$.

[2] S. MAJID, Cross products by braided groups and bosonization, J. Algebra 163, (1994), n 1, $165-190$.

[3] Ph. Nuss, M. WAmbst, Non-Abelian Hopf Cohomology, J. Algebra 312, (2007), nº 2, 733 754.

[4] Ph. Nuss, M. WAmbst, Non-abelian Hopf cohomology II - The general case, J. Algebra 319, (2008), $\mathrm{n}^{\circ} 11,4621-4645$.

[5] D. E. RADFORD, The structure of Hopf algebras with a projection, J. Algebra 92, (1985), n 2, $322-347$.

[6] J.-P. SERRE, Corps locaux, Troisième édition corrigée, Hermann, Paris (1968).

[7] J.-P. SERRE, Galois cohomology, Springer-Verlag, Berlin - Heidelberg (1997). Translated from Cohomologie galoisienne, Lecture Notes in Mathematics 5, Springer-Verlag, Berlin Heidelberg - New York (1973).

[8] Chr. SoulÉ, Les variétés sur le corps à un élément, Mosc. Math. J. 4 (2004), nº 1, 217-244, 312 .

[9] M. E. SweedleR, Cohomology of algebras over Hopf algebras, Trans. Amer. Math. Soc. 133 (1968), $205-239$.

[10] E. J. TAFT, The order of the antipode of finite-dimensional Hopf algebra, Proc. Nat. Acad. Sci. USA 68 (1971), nº 11, $2631-2633$.

[11] D. N. YetTeR, Quantum groups and representations of monoidal categories, Math. Proc. Camb. Phil. Soc. 108 (1990), nº 2, $261-290$. 